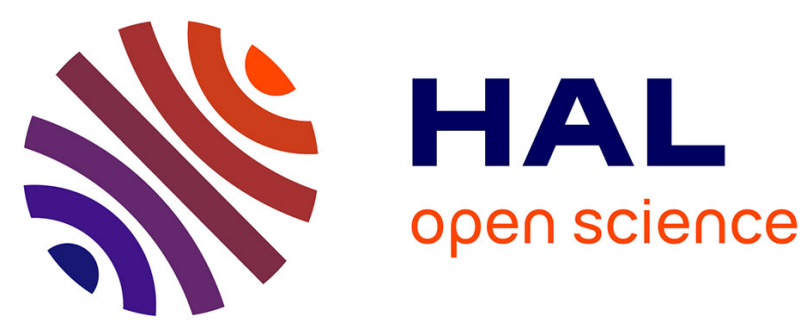

\title{
Transport of UDP-Rhamnose by URGT2, URGT4 and URGT6 modulates Rhamnogalacturonan-I length
}

Susana Saez Aguayo, Juan Pablo Parra-Rojas, Pablo Sepúlveda-Orellana, Jonathan Celiz-Balboa, Verónica Arenas-Morales, Christine Salle, Hernán Salinas-Grenet, Asier Largo-Gosens, H.M. North, Marie-Christine Ralet, et al.

\section{To cite this version:}

Susana Saez Aguayo, Juan Pablo Parra-Rojas, Pablo Sepúlveda-Orellana, Jonathan Celiz-Balboa, Verónica Arenas-Morales, et al.. Transport of UDP-Rhamnose by URGT2, URGT4 and URGT6 modulates Rhamnogalacturonan-I length. Plant Physiology, 2021, 185 (3), pp.914-933. 10.1093/plphys/kiaa070 . hal-03128745

\section{HAL Id: hal-03128745 \\ https://hal.inrae.fr/hal-03128745}

Submitted on 2 Feb 2021

HAL is a multi-disciplinary open access archive for the deposit and dissemination of scientific research documents, whether they are published or not. The documents may come from teaching and research institutions in France or abroad, or from public or private research centers.
L'archive ouverte pluridisciplinaire HAL, est destinée au dépôt et à la diffusion de documents scientifiques de niveau recherche, publiés ou non, émanant des établissements d'enseignement et de recherche français ou étrangers, des laboratoires publics ou privés.

$$
\text { Copyright }
$$


Transport of UDP-Rhamnose by URGT2, URGT4 and URGT6 modulates Rhamnogalacturonan-I length

\section{AUTHORS}

Susana Saez-Aguayo ${ }^{a}$, Juan Pablo Parra-Rojas ${ }^{a}$, Pablo Sepúlveda-Orellana ${ }^{a}$, Jonathan Celiz-Balboa $^{\mathrm{a}}$, Verónica Arenas-Morales ${ }^{\mathrm{a}}$, Christine Sallé ${ }^{\mathrm{b}}$, Hernán Salinas-Grenet ${ }^{\mathrm{a}}$, Asier Largo-Gosens ${ }^{\mathrm{a}}$, Helen M. North ${ }^{\mathrm{b}}$, Marie-Christine Ralet ${ }^{\mathrm{c} 1}$, and Ariel Orellana ${ }^{\mathrm{a} 1}$

${ }^{a}$ Centro de Biotecnología Vegetal, ${ }^{1}$ FONDAP Center for Genome Regulation, Facultad de Ciencias de la Vida, Universidad Andrés Bello, Santiago, Chile;

${ }^{b}$ Institut Jean-Pierre Bourgin, UMR1318, INRAE, AgroParisTech, Route de Saint Cyr, RD10, F-78026 Versailles Cedex, France;

' UR1268 BIA, INRAE, 44300, Nantes, France;

' In memory of Christine Sallé; we appreciate her collaborative work, her friendship, her happiness and her kindness.

${ }^{1}$ Senior Authors

Corresponding author:

Ariel Alejandro Orellana López, PhD

Avenida República 330, Santiago, RM 837-0146, Chile

(+562-26618628)

E-mail: aorellana@unab.cl

Short Title: URGT2, URGT4, and URGT6 modulate RG-I length

One-sentence summary: Three UDP-rhamnose/UDP-galactose transporters play different roles in the biosynthesis of mucilage and the lack of all three results in the production of shorter rhamnogalacturonan polymers and altered xylan domains.

\section{Author Contributions}

S.S-A., H.M.N., M.-C.R, and A.O. designed the research. S.S.-A., J.P.P-R., J.C., V.A., 
P.S., and A.L-G. performed the experiments. C.S. produced seeds for analyses. S.S.-A., J.P.P-R., A.L.-G., H.M.N., M.-C.R., and A.O. analyzed the data. S.S.-A., A.L-G., H.S-G., M.-C.R., H.M.N., and A.O. wrote the article.

\section{Abstract}

Rhamnogalacturonan-I biosynthesis occurs in the lumen of the Golgi apparatus, a compartment where UDP-Rhamnose and UDP-Galacturonic Acid are the main substrates for synthesis of the backbone polymer of pectin. Recent studies showed that UDP-Rha is transported from the cytosol into the Golgi apparatus by a family of six UDP-rhamnose/UDP-galactose transporters (URGT1-6). In this study, analysis of adherent and soluble mucilage of Arabidopsis thaliana seeds revealed distinct roles of URGT2, URGT4, and URGT6 in mucilage biosynthesis. Characterization of soluble mucilage polymer size showed shorter chains in the urgt2 urgt4 and urgt2 urgt4 urgt6 mutants, suggesting that URGT2 and URGT4 are mainly involved in RG-I elongation. Meanwhile, mutants in urgt6 exhibited changes only in adherent mucilage. Surprisingly, the estimated number of RG-I polymer chains present in urgt2 urgt4 and urgt2 urgt4 urgt6 mutants was higher than in wild-type. Interestingly, the increased number of shorter RG-I chains was accompanied by an increased amount of xylan. In the urgt mutants, expression analysis of other genes involved in mucilage biosynthesis showed some compensation. Studies of mutants of transcription factors regulating mucilage formation indicated that URGT2, URGT4, and URGT6 are likely part of a gene network controlled by these regulators and involved in RG-I synthesis. These results suggest that URGT2, URGT4, and URGT6 play different roles in the biosynthesis of mucilage, and the lack of all three affects the production of shorter RG-I polymers and longer xylan domains.

\section{Introduction}

The plant cell wall is a highly dynamic structure that surrounds the cell membrane and acts as a physical barrier, influencing plant development, cell morphology and also controlling external environmental cues (Verhertbruggen et al., 2013; Levesque-Tremblay et al., 2015; Rasool et al., 2017; Wolf et al., 2017). The primary 
plant cell wall is composed mainly of three classes of polysaccharides: cellulose, hemicelluloses, and pectin, which are organized in a complex network that can be restructured to allow cell elongation and differentiation, ensuring its functionality (Carpita and Gibeaut, 1993). It has been proposed that cellulose and hemicelluloses form a rigid network that provides the tensile strength of the plant cell wall, while pectin forms a gelatinous network implicated in cell-cell adhesion and the determination of wall porosity (Cosgrove, 2016). Cellulose and hemicelluloses are largely built of neutral sugars that are mainly connected by $\beta-1,4$ linkages. Pectin is the most complex polysaccharide in the plant cell wall, characterized by its high content of acidic sugars, mainly composed of galacturonic acid (GalA) residues connected by $\alpha-1,4$ linkages (Mohnen, 2008). Four domains characterize pectins, three of them being made of a backbone of GalA residues, which can be unbranched (homogalacturonan/HG), substituted with xylose (Xyl) residues (xylogalacturonan/XGA), or decorated with a conserved set of complex side chains (rhamnogalacturonan-II/RG-II) (Mohnen, 2008). Rhamnogalacturonan-I (RG-I) differs from these three pectin domains because its backbone is not only composed of GalA residues but consists of a repeated $\alpha$-D-1,4-GalA- $\alpha-L-1,2-R$ ha (rhamnose) disaccharide. The Rha residues in the RG-I backbone can be substituted with side-chain structures, including linear $\beta$-1,4-linked -galactan and $\alpha-1,5$-linked -arabinan or arabinogalactans containing both galactose (Gal) and arabinose (Ara) units (Atmodjo et al., 2013). To date, the in vivo structure of pectin macromolecules has not been well defined due to the difficulty of extracting them as an intact structure without altering their natural form (Atmodjo et al., 2013). Over the last two decades, the mucilage pocket synthesized in the Arabidopsis (Arabidopsis thaliana) seed coat has been used to study in vivo pectin biosynthesis and structure (Western et al., 2000; Macquet et al., 2007a; Voiniciuc et al., 2015). Mucilage consists of a jelly-like structure mainly composed of pectin (Western et al., 2000; Macquet et al., 2007a; Voiniciuc et al., 2015c) and has a highly organized structure constituted of a soluble (SM) and an adherent layer (AM). Both layers are composed mainly (more than $90 \%$ ) of unbranched RG-I (Macquet et al., 2007a). The outer soluble mucilage layer can be removed easily upon water imbibition. In addition to RG-I, it contains trace amounts of neutral sugars such as Ara, Xyl, mannose (Man), Gal, and glucose (Glc) (Macquet et al., 2007a; 
Voiniciuc et al., 2015 b and c; Ralet et al., 2016). In contrast, the inner adherent layer is firmly attached to the seed surface. It can be detached from the seed by digestion with rhamnogalacturonan hydrolase (Macquet et al., 2007a), by harsh chemical treatment (Huang et al., 2011; Walker et al., 2011), by high-speed mechanical agitation (Voinicuic et al., 2015c) and by ultrasonic treatment (Zhao et al., 2017). In addition to RG-I, the adherent mucilage contains small amounts of cellulose, arabinan, galactan, galactoglucomannan, and homogalacturonan (Macquet et al., 2007a, Voinicuic et al., 2015b; Ralet et al., 2016).

In recent years, several genes have been described that encode for glycosyltransferases (GT), which participate in the synthesis of mucilage polysaccharides. This is the case of IRREGULAR XILEM14 (IRX14) and MUCILAGE RELATED-21 (MUM5/MUCl21), putative xylosyltransferases (XylT), which have been proposed to be responsible for the elongation and branching of xylan domains that may anchor mucilage RG-I to cellulose microfibrils (Voiniciuc et al., 2015c; Hu et al., 2016; Ralet et al., 2016). GALACTURONOSYLTRANSFERASE-LIKE 5 (GATL5) and MUCILAGE-RELATED 70 (MUCI70) have been described as putative galacturonosyl transferases involved in RG-I biosynthesis (Voiniciuc et al., 2018; Fabrissin et al., 2019). Also, GALACTURONOSYLTRANSFERASE 11 (GAUT11) is a homogalacturonan galacturonosyltransferase that synthesizes HG (Kong et al., 2013; Voiniciuc et al., 2018; Fabrissin et al., 2019). Finally, RG-I RHAMNOSYLTRANSFERASE 1 (RRT1) was recently described as a rhamnosyl transferase participating in RG-I elongation (Takenaka et al., 2018). Nucleotide sugars, the donor substrates used by all these Golgi-localized GTs required for RG-I, xylan, and HG polymerization, are synthesized in a process that could be performed in the cytosol and within the Golgi apparatus (Reyes and Orellana., 2008; Temple et al., 2016). UDP-GalA and UDP-Xyl could be synthesized both in the cytosol and in the Golgi Apparatus, whereas UDP-Rha is formed exclusively in the cytosol. This implies that cytosolic nucleotide sugars (such as UDP-Rha) need to be transported into the lumen of the Golgi apparatus for their incorporation into a nascent polymer (Reyes and Orellana., 2008; Temple et al., 2016). To cope with this topological problem, a family of proteins called Nucleotide Sugar Transporters (NSTs) located in the Golgi membrane, are responsible for the transport of 
UDP/GDP-sugars from the cytosol to the Golgi lumen, supplying the substrates needed for polysaccharide biosynthesis to the GTs (Reyes and Orellana, 2008; Orellana et al., 2016; Temple et al., 2016). In Arabidopsis thaliana, 44 genes encoding NSTs, similar to those encoding plastid triose phosphate translocators (TPTs), together form an NST gene family of 51 members (Knappe et al., 2003; Rautengarten et al., 2014). Recently, UDP-RHAMNOSE/UDP-GALACTOSE TRANSPORTER 2 (URGT2) and UDP-URONIC ACIDS TRANSPORTER 1 (UUAT1) transporters were characterized as a UDP-rhamnose/UDP-galactose transporter and a UDP-uronic acid transporter, respectively, involved in mucilage synthesis. Indeed, urgt2 and uuat1 mutants had modified mucilage with lower amounts of Rha and GalA in the SM layer, suggesting that accumulation of mucilage RG-I is affected in these mutants (Rautengarten et al., 2014, Saez-Aguayo et al., 2017; Parra-Rojas et al., 2019).

URGT2 is part of the characterized clade of the nucleotide sugar transporter family composed of 6 members (URGT1-6), which are all able to transport UDP-Rha and UDP-Gal in vitro (Rautengarten et al., 2014). The weak phenotype of the urgt2 mutant, with a decrease of only $20 \%$ of mucilage sugar contents, suggests the participation of other URGTs in the process of mucilage production in seed coat epidermal cells. Here we show that the six URGT (1-6) members are expressed in the seed coat at the time when mucilage polymers are being synthesized, meaning that all six members could contribute to this process. To determine which of these URGT genes are required for mucilage production, mutants in each gene were obtained and analyzed for defects in mucilage production. The results revealed that urgt2-2, urgt4-2, and urgt6-1 seeds exhibit a modest, though significant decrease in SM amount, suggesting the participation of all three transporters in the synthesis of mucilage RG-I. Detailed biochemical analysis coupled with physicochemical analysis of the soluble mucilage extracted from the URGT2, URGT4, and URGT6 single, double and triple mutant seeds showed that urgt2-2 urgt4-2 and the triple urgt mutant have shorter RG-I polymers with a concomitant increase in the number of RG-I chains. Furthermore, the monosaccharide composition of this mucilage fraction had an increased Xyl content, which was directly proportional to the increase in the number of RG-I chains observed in urgt mutants, suggesting a coordinated synthesis of the RG-I backbone and xylan side-chains. 
Additionally, the single, double, and triple urgt mutants exhibited different levels of AM density determined by FITC-dextran staining, suggesting that URGT2, URGT4, and URGT6 participate in the adherent mucilage production independently. Finally, RT-qPCR analysis in developing seeds from the triple mutant, assessing some genes likely involved in RG-I biosynthesis, exhibited a lower level of transcripts at the onset of mucilage formation. However, some transcripts showed higher levels at later stages, suggesting that genes involved in RG-I and xylan production are misregulated in the triple mutant, perhaps to compensate for changes in mucilage production. These results show the importance of URGT2, URGT4, and URGT6 in the formation of mucilage RG-I and describe the association of NSTs with the regulation of the number of RG-I molecules produced and their length.

\section{Results}

\section{urgt2, urgt4, and urgt6 mutant lines exhibit reduced rhamnose content in soluble mucilage}

The relative transcript abundance of URGT genes was determined in a range of plant organs throughout development by RT-qPCR, normalizing the expression against two reference genes (Figure 1 and Supplemental Figure 1). Most of the URGTs were ubiquitously expressed in planta, suggesting an essential and redundant role during plant development. To determine the contribution of URGTs to RG-I synthesis in mucilage, first, we examined in seeds URGTs RNA transcript accumulation during the developmental stages at which mucilage polysaccharides are deposited in the apoplasm. Hence, their expression was analyzed at 6, 8, 10, and 12 Days After Pollination (DAP) (Figure 1). Except for URGT5, all the URGT members were strongly expressed during these developmental stages. Also, URGT1, URGT2, and URGT4 expression peaked at 8 to 10 DAP, which corresponds to the stages when polysaccharide accumulation is the strongest in the seed coat epidermal cells. Microarray data, from developing seed tissue (eFP browser) at stages when mucilage is

produced show that among the six URGT members, URGT2, URGT4, and URGT6 exhibit strong expression at the linear cotyledon stage (Supplemental Figure 2) (Winter 
et al., 2007; Bassel al., 2008). These expression profiles suggested that, in addition to the previously characterized URGT2 (Rautengarten et al., 2014; Parra-Rojas et al., 2019), URGT1, URGT4, and URGT6 could be involved in the biosynthesis of mucilage pectin. Nevertheless, the participation of URGT3 and URGT5 could not be excluded because they also showed expression in the seed coat (Figure 1 and Supplemental Figure 2). Therefore, to further determine which of the six URGT genes impact mucilage formation, we screened at least one homozygous insertional mutant line for each URGT, and the content of Rha was assessed in the outer layer of seed mucilage (Figure 2).

The results showed lower levels of Rha in urgt2, urgt4, and urgt6. The analysis of GalA contents also indicated lower levels of this monosaccharide in mucilage (Table 1; Supplemental Table 1); thus, these results suggested that transporters encoded by these three genes play a role in the synthesis of mucilage RG-I. No obvious phenotype was observed in single, double, and triple mutant plants. Then, we performed a thorough analysis of every single mutant, and no differences between wild-type and the mutants were observed for germination rate and rosette size. Cotyledons were normal in all mutants (Supplemental Figure 3A); however, urgt2-2 and urgt4-2 seedlings exhibited roots that were $7.7 \%$ and $21.8 \%$ shorter than wild-type at day 7 of growth (Supplemental Figure 3B). Even though URGT4 is more expressed in roots than URGT2, it is hard to provide an explanation for this result since we have no information about the role that URGT4 and URGT2 are playing in root cells; hence, this finding needs further analysis in future work.

\section{urgt2, urgt4, and urgt6 exhibit different soluble and adherent mucilage phenotypes}

Our results suggested that URGT4 and URGT6 contribute to the biosynthesis of mucilage RG-I; thus, to confirm these results, a second mutant allele was selected for each of these genes and further analyzed (Supplemental Figure 4). RT-qPCR analyses (Supplemental Figure 5) revealed that urgt4-2, urgt4-3, and urgt6-2 mutant lines are knock-down mutants with reductions of $65 \%, 57 \%$ and $43 \%$ of URGT4 and URGT6 
expression, respectively. In contrast, no accumulation of URGT6 transcripts was observed in urgt6-1. The impact of each mutation on mucilage biosynthesis was assessed by measuring GalA content (Figure 3 and Supplemental Table 2A). A slight decrease in GalA was observed in SM and AM from urgt2-1, urgt2-2, urgt4-2, and urgt4-3 mutants, but no significant changes were observed for urgt6-1 and urgt6-2. Furthermore, to analyze the RG-I content and distribution in the adherent mucilage, whole-mount immunolabelling was carried out using the INRA-RU1 antibody, which recognizes RG-I with at least six Rha-GalA disaccharide repeats (Ralet et al., 2010). Reduced labeling (green fluorescence) was observed in all mutant lines (Supplemental Figure 6), suggesting that URGT2, URGT4, and URGT6 are required to generate RG-I epitopes in the AM. To confirm that these genes are responsible for the observed phenotypes, we carried out the molecular complementation of the urgt2-1, urgt4-2, and urgt6-1 mutant lines by transforming them with the constructs proURGT2:URGT2:GFP, proURGT4:URGT4:GFP and proURGT6:URGT6:GFP, respectively. For each mutant, three independent homozygous transformant lines containing a single insertion of the construct were selected. We verified the rescue of each URGT mutant line by analyzing the expression of the corresponding URGT by RT-qPCR (Supplemental Figures 7B, 8B, 9B).

Results showed the recovery to wild-type or higher levels of URGT gene expression for all urgt2-2, urgt4-2, and urgt6-1 transformants, indicating molecular rescue in these lines. The immunolabelling of RG-I epitopes on AM of transformants using the INRA-RU1 antibody revealed a recovery of the RG-I labeling (Supplemental Figures $7 \mathrm{~A}-\mathrm{C}, 8 \mathrm{~A}-\mathrm{C}, 9 \mathrm{~A}-\mathrm{C})$, confirming that URGT2, URGT4, and URGT6 play a role in the biosynthesis of RG-I.

\section{Analysis of the contribution of URGT2, URGT4, and URGT6 to mucilage-RG-I biosynthesis in double and triple mutants}

To further investigate the contribution of URGT2, URGT4, and URGT6 to mucilage RG-I biosynthesis, crosses were carried out using urgt2-2, urgt4-2 and urgt6-1; thus, different combinations of double mutants and a triple URGT mutant were obtained. Then, the 
expression of the URGT genes in the double and triple mutants was evaluated by RT-PCR and by RT-qPCR (Supplemental Figure 10 and Supplemental Figure 11). No significant changes in expression of the different URGTS (URGT1, URGT2, URGT3, URGT4, URGT5 and URGT6) were observed in any the mutants (Supplemental Figure 10 and Supplemental Figure 11). As we described above, urgt2-2 and urgt6-1 were knock-outs, and they were absent in all the combinations that included them; however, both urgt4 alleles were knock-downs, with approximately $25-30 \%$ of WT URGT4 expression observed in the double and triple mutants (Supplemental Figure 10). To determine the impact on mucilage polysaccharide production in the double and triple mutants, we measured the GalA contents of both AM and SM layers. The results showed that urgt2-2 urgt4-2 and the triple urgt2-2 urgt4-2 urgt6-1 mutants were the most affected in mucilage production, with decreases in total mucilage GalA contents of $29.1 \%$ and $36.1 \%$, respectively, compared to wild-type. These results showed an additive effect on the biosynthesis of mucilage pectin in the double and triple mutants (Figure 4 and Table 1).

To gain information about other changes that may occur in the mucilage from the different mutants, a detailed analysis of the monosaccharide composition was performed on the SM and AM layers, as well as demucilaged seeds (Table 1). No significant differences in sugar content were observed among demucilaged seeds from all the mutants and wild-type, confirming that the differences observed were specific to mucilage polysaccharide production by the epidermal cells of the seed coat. Similar reductions to those observed for GalA were observed for Rha contents in both SM and AM from the different mutants (Table 1).

Interestingly, a significant increase in the content of Xyl was observed in the SM from all the double mutants and the triple mutant: urgt2-2 urgt4-2 (50.8\%) urgt2-2 urgt6-1 (27.1\%), urgt4-2 urgt6-1 (23.7\%) and the triple mutant (130.5\%). We also observed small variations in the contents of Gal, Man, and Glc in SM from some single, double, and triple mutant lines (Table 1). Furthermore, small differences in Ara, Gal, and Glc contents from AM were also observed in single, double, and triple mutant lines. Comparison of total sugar contents for AM and SM from the different mutants showed 
significant reductions in urgt2-2, the three double mutants and the triple mutant with the strongest defect observed in the urgt2-2 urgt4-2 and the triple mutant (Table 1).

\section{urgt2, urgt4, and urgt6 mutants have modified soluble mucilage macromolecular characteristics}

The decrease in GalA and Rha contents in the mutants suggests that RG-I may be affected in its molecular features. Therefore, to assess potential changes of RG-I in the mutants, polymers from the soluble mucilage layer were analyzed using high-performance size-exclusion chromatography (HP-SEC) coupled with intrinsic viscosity measurements. Since SM is composed of mainly linear RG-I chains, these measurements provide a good estimation of the length of the RG-I polymer (Macquet et al., 2007a; Kong et al., 2013; Williams et al.,2020). Intrinsic viscosity values for urgt2 and urgt4 mutants showed that RG-I polymers were shorter, with a reduction in intrinsic viscosity of $24.2 \%, 28.6 \%, 9.3 \%$, and $12.3 \%$ compared to wild-type for urgt2-1, urgt2-2, urgt4-2, and urgt4-3, respectively (Supplemental Table 2B). A slight, but not significant, decrease in the intrinsic viscosity was observed for urgt6-1 and urgt6-2 mutant lines compared to wild-type.

To determine the potential redundancy of the three URGT members in determining the length of RG-I polymers, the intrinsic viscosity of the outer mucilage layer was analyzed in the different double mutant combinations and the triple urgt mutant (Figure 5A). A significant decrease in the intrinsic viscosity was observed in all double and triple urgt mutants. However, the strongest reductions were observed in the urgt2-2 urgt4-2 and the triple mutants, where the intrinsic viscosity decreased by $59.7 \%$ and $66.6 \%$, respectively (Figure 5A).

We obtained an intrinsic viscosity value of the order of $6 \mathrm{dL} / \mathrm{g}$ for the principal polymer population of wild-type Col-0, in good agreement with the literature (Macquet et al., 2007a, Sullivan et al., 2011; Griffiths et al., 2015., Wiliams et al., 2020). Considering the average mass of approximately $600 \mathrm{kDa}$ in the wild-type (Macquet et al., 2007, Sullivan et al., 2011; Griffith et al., 2016., Williams et al., 2020) and the fact that the [Rha-GalA] subunit has a molecular mass of $322 \mathrm{~g} / \mathrm{mol}$, the number of [Rha-GalA] subunits/dimers 
in an RG-I polymer from SM can be estimated as approximately 1845 for the wild-type. Meanwhile, for the urgt2-2 urgt4-2 and the triple mutants, we estimated that the RG-I molecules are composed of 756 and 629 [Rha-GalA] dimers, respectively (Supplemental Table 3). In addition, by using the amount (in moles) of Rha and GalA, plus the Avogadro constant and the calculated number of [Rha-GalA] dimers, we were able to estimate the total number of RG-I molecules present in the SM for each mutant line and the wild-type (Figure 5B; Fabrissin et al., 2019). Higher numbers of RG-I chains were calculated for urgt2-2 urgt4-2 and the triple urgt mutant, compared to the wild-type, with increases of $62 \%$ and $109 \%$, respectively (Figure 5B).

\section{RG-I branching and xylan length appear to be altered in the outer mucilage of the triple urgt mutant}

Among the differences observed in sugar content, the substantial increase in xylose observed in the outer mucilage extracted from dry seeds of double and triple mutants was particularly noteworthy (Table 1) and suggested an alteration in xylose-enriched polysaccharides in these mutants. Recent studies have shown that mucilage RG-I is accompanied by xylan domains present in a tight proportionality to the number of constituent GalA-Rha disaccharides and which are required for its adherence to cellulose microfibrils and the seed surface (Ralet et al., 2016; Voiniciuc et al., 2018; Fabrissin et al., 2020). To understand the xylose increase in the mutants, we first calculated the ratio between the number of $X y l$ residues and the number of RG-I molecules in the urgt mutant lines (Figure 6). The analysis predicted approximately 67 Xylose residues for each RG-I molecule, in both wild type and the different urgt mutant lines, indicating that the proportion of Xyl per RG-I is not affected in their mucilage. To investigate further the structure of RG-I and xylan domains, a glycosyl linkage analysis of WT and triple mutant SM was performed. Xylan chains are formed from 1,4-linked xylose units that can be branched at O-2 (Table 2, 1,2,4-Xyl) and finish with a terminal-xylosyl (Table 2, Xyl-t). The ratio of 1,2,4-Xyl/1,4-Xyl/Xyl-t in triple mutant was $0.3 / 3 / 1$ vs $0.4 / 0.3 / 1$ in the wild-type (Table 2 ) suggesting that xylan branches in the mutant are longer than those of wild-type (Voiniciuc et al., 2018; Fabrissin et al., 2019). An indication of the number of RG-I branches can also be deduced from the ratio of 
rhamnosyls with side-chain linkages (1,2,4-Rha) vs the number of rhamnosyls present in the RG-I backbone (1,2-Rha) and terminal rhamnosyls (Rha-t). In the triple mutant, this ratio was $0.09 / 23.1 / 1$ vs $0.15 / 22.2 / 1$ in the wild-type, suggesting that the RG-I macromolecules in mutant mucilage are less branched that those of wild-type (Voiniciuc et al., 2018; Fabrissin et al., 2019).

\section{Combination of urgt2, urgt4, and urgt6 mutations has different effects on adherent mucilage structure}

To evaluate the contribution of URGT2, URGT4 and URGT6 in the formation of the inner AM layer, whole-mount immunolabelling of RG-I epitopes in the AM released from mature seeds of urgt mutants was performed and compared to wild-type using the INRA-RU1 antibody (Ralet et al., 2010, Figure 7). As described above, reduced labeling (green fluorescence) was observed in urgt2-2, urgt4-2, and urgt6-1 in comparison to wild-type. In contrast, more labeling and a more intense signal were observed in the double (urgt2-2 urgt4-2 and urgt2-2 urgt6-1) and triple urgt mutant seeds, suggesting that the epitopes were either more abundant or more accessible in these mutants. No significant differences in labeling were observed in the urgt4-1 urgt6-1 double mutant in comparison to the wild-type.

Increased antibody accessibility could be due to a modified density of the AM. To evaluate changes in the porosity of the AM, we used dextran molecules labeled with fluorescein isothiocyanate (FITC) (Willats et al., 2001; Voiniciuc et al., 2015). Using 70-kD FITC-dextran, we observed a black halo in the AM of wild-type seeds, a result that is likely obtained because the inner mucilage capsule, composed of densely packed polysaccharides, prevents the diffusion of FITC-Dextran (Figure 8A). While the AM of the urgt6-1 mutant exhibited a similar feature to the wild-type, the other urgt single, double and triple mutants exhibited an altered FITC-dextran penetration. The results of measuring the width of the halo in comparison to the size of the seed (Figure $8 \mathrm{~B}$ ) or the intensity of the fluorescence within the halo (measured as pixels per $\mu \mathrm{m}^{2}$, Figure $8 \mathrm{C}$ ), allowed us to classify the mutants into three phenotypic groups. The urgt2-2 mutant was unique in having a similar fluorescence intensity to wild-type, but a smaller 
mucilage halo.

In contrast, in the urgt4-2 and urgt4-2 urgt6-1 mutants, the mucilage halo was similar in size to the wild-type, but the fluorescence intensity was higher, suggesting that the AM is less densely packed. Finally, urgt2-2 urgt4-2, urgt2-2 urgt6-1, and the triple mutant had smaller halos and higher fluorescence intensities, suggesting that the AM from these mutants was more porous.

\section{RRT1, GATL5, MUCI21 and UXT3 expression are differentially accumulated in the triple urgt mutant line}

Given the changes in RG-I and Xyl, we wondered whether some of the genes involved in their synthesis exhibited altered expression in the triple mutant. Previously, it was demonstrated that $R R T 1$ are involved in mucilage RG-I biosynthesis (Kong et al., 2013; Takenaka et al., 2018). Additionally, MUM5/MUCl21 encodes for a putative xylosyltransferase involved in the modification of xylan present in mucilage (Voiniciuc et al., 2015; Ralet et al., 2016). Besides, UDP-XYLOSE TRANSPORTER 3 (UXT3) encodes for a UDP-xylose transporter involved in xylan biosynthesis, which is highly expressed in the seed coat integument when mucilage polysaccharides are produced (Le et al., 2010; Ebert et al., 2015). To determine if the expression of these genes was misregulated in the triple mutant, we compared their transcript accumulation in developing seeds from 5 DAP to 12 DAP (Figure 9). In the wild-type, RRT1, GATL5, and MUCl21 had a peak of expression at 8-9 DAP, with lower levels at the beginning (5-7 DAP) and the end (11-12 DAP) of seed development (Figure 9). This pattern of expression contrasted with the expression profile of the UXT3 gene, which was higher at early developmental stages (5-8 DAP) than later stages (9-12 DAP). In the triple urgt mutant, similar profiles to those of wild-type were observed for all four genes; however, the expression level for RRT1 was lower at early stages of seed development (5-6 DAP) and higher at later stages of development (10-12 DAP) in comparison to the wild-type (Figure 9A). GATL5 transcript abundance in the triple mutant was lower from 5 to 9 DAP, and then higher at 10-11 DAP (Figure 9B). Finally, expression of MUM5/MUCI21 was less abundant at 6 DAP and more abundant at 10 and 12 DAP, whereas, UXT3 
transcripts were less abundant at 5, 6, 10, and 11 DAP, and increased at 12 DAP (Figure 9C and 9D). We concluded that the genes analyzed tend to have a lower expression at the beginning and an increase towards the end of seed development in the triple mutant compared to wild-type.

URGT2, URGT4, and URGT6 are dissimilarly orchestrated by GL2, TTG2, MYB61, and MUM1

The transcription regulators GLABRA2 (GL2), TRANSPARENT TESTA GLABRA2 (TTG2), MYB61 and LEUNIG_HOMOLOG (LUH)/MUCILAGE-MODIFIED 1 (MUM1) have been previously described to be part of the complex regulatory network that controls the synthesis of mucilage polysaccharides in the Arabidopsis seed coat (reviewed by Golz et al., 2018). Therefore, if URGT2, URGT4, and URGT6 are involved in mucilage biosynthesis, their expression could be regulated by these transcription factors. All four regulators are highly expressed in developing seeds at 8 DAP (Supplemental Figure 13, so to assess whether these transcription factors are involved in regulating the expression of URGT2, URGT4, and URGT6, their transcript abundance was determined in developing seeds of luh/mum1, gl2, ttg2 and myb61 mutants at 8 DAP. URGT2 transcript levels were markedly reduced in all four mutants compared to wild-type (Figure 10). Similar, but more modest reductions were also observed in URGT4 transcript levels in all the mutants. In contrast, URGT6 expression levels were only modified in the luh/mum1 mutant, where levels were slightly higher. These results indicate that the expression of URGT2 with URGT4 is positively regulated by GL2, TTG2, MYB61, and MUM1, while MUM1 represses URGT6 expression.

\section{Discussion}

\section{URGT2, URGT4, and URGT6 contribute to mucilage polysaccharide synthesis}

A family of six URGT genes encodes Golgi-localized transporters that transport UDP-Rha and UDP-Gal in vitro (Rautengarten et al., 2014). These are the only NST genes reported to date capable of transporting UDP-Rha; thus, they likely play a role in 
supplying the substrate for the biosynthesis of Rha-containing polysaccharides. Mucilage is highly enriched in rhamnogalacturonan-l; therefore, it is a suitable system to analyze the contribution of each URGT to the biosynthesis of this polysaccharide. Previous reports from our laboratory already showed that URGT2 plays a role in this process (Rautengarten et al., 2014; Parra-Rojas et al., 2019); however, seeds from urgt2 only had a $20 \%$ decrease in mucilage. This partial decrease raised the question: which other URGT members participate in the biosynthesis of RG-I or could functionally compensate for the impact of knocking out URGT2?

Since all URGTs are expressed in developing seeds, we assessed the sugar content in mucilage in mutants of all six URGT genes and found reductions in Rha and GalA in SM for urgt2, urgt4 mutants (Figure 1 and Figure 2). In contrast, Rha and GalA contents in SM were not affected in the urgt6-1 mutant. However, a small but significant decrease in Rha content in AM was observed, indicating a subtle effect on RG-I production in of RG-I epitopes in the inner mucilage was less intense in urgt2, urgt4, and urgt6, suggesting that while absolute levels of polymer sugars were not strongly affected in the mutants, RG-I present in AM was somehow altered (Figure 7). Based on these results, we concluded that URGT2 makes a significant contribution to UDP-Rha transport for the synthesis of both SM and AM layers, whereas URGT6 seems to play a more important role in AM production. Finally, URGT4 contributes to the synthesis of both SM and AM layers but, since we could only analyze knock-down mutants with a residual expression of around $25 \%$, it was not possible to define the precise contribution of this gene to mucilage biosynthesis (Supplemental Figure 5). We found no evidence for URGT1, URGT3, and URGT5 playing a role in the biosynthesis of RG-I in seeds, indicating that in wild-type genotype conditions, not all URGTs are supplying the UDP-Rha needed for the biosynthesis of this polymer.

\section{Mutation of URGT2, URGT4 or URGT6 had different impacts on mucilage production}

As the urgt2, urgt4, and urgt6 single mutants did not have a strong impact on mucilage composition, the analysis of double and triple urgt mutant combinations was carried out 
to gain information about the relative contribution of each URGT to the production of mucilage RG-I polymers. The urgt2-2 urgt4-2 plants showed the strongest decrease in Rha and GalA in mucilage from all possible combinations that produced double mutants (Figure 4 and Table 1). Although the triple mutant exhibited a similar reduction, a greater decrease of Rha in the AM layer was observed in the triple mutant. These results confirmed the participation of all three URGTs in the synthesis of RG-I secreted into the mucilage pocket, with URGT2 and URGT4 playing a more critical role than URGT6. Rautengarten et al., (2014) reported that the URGT family are transporters of UDP-Rha and UDP-Gal based on their in vitro activity. Moreover, it has been proposed that RG-I is synthesized by the coordinated action of a rhamnosyltransferase and a galacturonosyltransferase, acting one after the other, adding Rha and GalA onto a growing RG-I chain (Takenaka et al., 2018). Therefore, the reduction in GalA observed in urgt single, double and triple mutants is a direct consequence of the defective RG-I synthesis due to reduced availability of UDP-Rha, whose transport into the Golgi is diminished. A similar phenotype was observed in the URONIC ACID TRANSPORTER mutant, uuat1, where the GalA reduction caused by the lack of UUAT1 function was accompanied by a decrease in Rha amounts in mucilage (Saez-Aguayo et al., 2017). In the same line, mutants of the putative and proven galacturonosyltransferases GATL5 and GAUT11, respectively, also exhibit a reduction in Rha and GalA contents (Kong et al., 2013 and Voiniciuc et al., 2018). Recently, the rhamnosyltransferase RRT1 was characterized, and mutants defective in that enzyme had reductions in both Rha and GalA contents (Takenaka et al., 2018). All these results confirm that any impairment in the incorporation of any of the two sugars that are part of the RG-I backbone produces a reduction in RG-I synthesis and a concomitant decrease in both Rha and GalA amounts in mucilage.

A shortage in the supply in UDP-Rha should lead to alterations in RG-I structure, and our results showed that polymers from soluble mucilage were shorter in the urgt2-2 mutant in comparison to wild-type. Polymer size was reduced even further in the double mutant urgt2-2 urgt4-2 and the triple mutant (Figure 5). These results provide evidence that the mutation of URGTs impaired the elongation of RG-I molecules. When RG-I 
polymer length was plotted against the number of RG-I molecules for each genotype, we could observe that RG-I length and molecule number content of the soluble mucilage layer are tightly proportional (Supplemental Figure 12). Indeed, we found that urgt2-2 urgt4-2 contained around 30\% more RG-I molecules than wild-type, with almost twice the number of polymers in the triple mutant. These results suggest that the reduction in RG-I synthesis due to the mutation of these URGTs leads to the synthesis of more, but shorter, RG-I molecules. Also, there is less UDP-Rha available for RG-I biosynthesis in the URGT mutants; therefore, elongation terminates prematurely, followed by subsequent reinitiation of a new polymer and multiple reiterations of this process.

\section{The proportion of xylose to RG-I molecules is maintained in soluble mucilage of urgt mutants, but the structure of xylan and RG-I chains are altered}

Previous studies have demonstrated that mucilage contains xylan domains that mediate the anchoring of RG-I molecules to cellulose microfibrils on the seed surface (Ralet et al., 2016). The efficient synthesis of these RG-I domains was recently shown to require MUCI70, which encodes a putative galacturonosyltransferase (Voiniciuc et al., 2018; Fabrissin et al., 2019). Combining a mutant in this gene and irx14, which is defective for a putative xylan synthase, led to stronger reductions in RG-I production than in the muci70 single mutant, showing that coordinated synthesis of $\mathrm{RGI}$ and xylan is essential for mucilage production (Voiniciuc et al., 2018; Fabrissin et al., 2019). Furthermore, reduced Rha and GalA contents in muci70 mucilage were associated with a strong increase in Xyl contents (Voiniciuc et al., 2018; Fabrissin et al., 2019). This chemotype is reminiscent of that observed here for the urgt double and triple mutants, where Xyl contents were increased in the SM of urgt2-2 urgt4-2 and triple mutants. In all urgt mutant genotypes, the ratio of $\mathrm{Xyl}$ residues per RG-I molecule was constant at approximately $67: 1$, the same as observed in wild-type. This result indicates that a change in the number of RG-I polymers and their length does not impact the proportion of Xyl to RG-I (Figure 6). Linkage analysis of the SM from the triple urgt mutant suggested a decrease in the branching of the RG-I backbone with a strong increase in 
the proportion of $1,4-X y l$, which forms linear xylan (Table 2). Based on the data collected for the triple mutant and the wild-type, we propose that the triple urgt mutant contains twice the number of RG-I molecules, which are three-times shorter than those present in wild-type mucilage. Linkage analysis data indicated that even though the proportionality between Xyl and RG-I polymers is maintained, the RG-I polymer is less substituted in the triple urgt mutant, and the structure of xylan domains might be different with longer xylan chains. However, these analyses should be verified by independent molecular weight determination techniques such as GPC-MALLS, after isolation of xylan moieties. These results are similar to those found in muci70 mucilage where shorter RG-I polymers were associated with altered xylan structure (Fabrissin et al., 2019), and reinforce the hypothesis proposed by Ralet et al. (2016) that a covalent link could exist between RG-I and xylan. Further experimental evidence is required to prove that xylan is covalently attached to RG-I; therefore, it cannot be excluded that RG-I length and branching influence the structure of free xylan domains or xylan attached to polymers in an AGP proteins (Tan et al., 2013).

\section{The role of URGTs in adherent mucilage structuration}

Increased labeling of AM was observed with an antibody against RG-I in double and triple mutant combinations of urgt2, urgt4, and urgt6, despite AM Rha and GalA sugar contents being reduced (Table 1; Figure 7). Analysis of the AM permeability using 70kD FITC-dextran showed that while the halo of AM around double and triple mutant seeds was smaller, its permeability was also increased compared to that of wild-type (Figure 8). These results indicated that increased labeling with INRA-RU1 could be due to a looser network, allowing access of the antibody to a larger number of epitopes within the AM. Also, it was described that INRA-RU1 binds to, at least, six unsubstituted Rha-GalA repeats (Ralet et al., 2010), and a higher permeability may facilitate the access to the epitopes. Interestingly, while differences in AM width corresponds well with differences in sugar contents, this appeared to be independent of permeability (Figure 8B compared to $\mathrm{C}$ ). This contrast was evident when comparing the urgt4 mutant and wild-type, or urgt2urgt4 compared to the triple mutant, where mucilage halos were more permeable, despite having equivalent widths and sugar contents. These results 
indicated that the regulation of RG-I polymer synthesis through the availability of UDP-Rha provided by URGTs impacts the interactions between the resulting polymers and the structure of AM.

\section{Genes involved in mucilage synthesis are misregulated in the triple urgt mutant}

To determine whether regulatory feedback mechanisms were initiated in response to the deficit of UDP-Rha, the expression of four genes, RRT1, GATL5, MUM5/MUCI21, and UXT3 was examined in developing seeds between 5 and 12 DAP. RRT1, GATL5 and MUM5/MUCl21 have demonstrated roles in mucilage synthesis (Kong et al., 2013, Voinicuic et al., 2015b; Takenaka et al., 2018). UXT3, encodes for a UDP-Xyl transporter, strongly expressed in the seed coat integument, but no mucilage phenotype has been reported (Winter et al., 2007; Ebert et al., 2015). Only GATL5 expression was reduced in the triple mutant during the stages of seed development when mucilage is actively synthesized (7-9 DAP). GATL5 encodes a putative galacturonosyl transferase that may participate in $H G$ domain synthesis. Still, it has also been implicated in determining RG-I length because gat/5 mutants produce longer mucilage RG-I molecules compared to wild-type (Kong et al., 2013). Interestingly, both GATL5 and $R R T 1$ were expressed at higher levels during the later stages of seed coat epidermal cell differentiation (10-12 DAP), when mucilage deposition is drawing to an end in wild-type. RRT1 is a rhamnosyltransferase explicitly involved in the synthesis of RG-I (Takenaka et al., 2018). In the triple urgt mutant, $R R T 1$ transcripts are accumulated at later stages of mucilage deposition. This difference from wild-type could be due to positive feedback regulation from reduced RG-I production or low UDP-Rha substrate levels. Similarly, expression of MUM5/MUCl21, which encodes a putative xylosyltransferase and is required to incorporate the first $\mathrm{Xyl}$ on Rha before xylan elongation by IRX14 takes place (Voiniciuc et al., 2015; Ralet et al., 2016), was also expressed at higher levels at 10 and 12 DAP. Both UXT3 and MUCI21 expression levels were decreased at the beginning of seed development. The former also showed a small decrease later during seed development. Together these results showed that the triple urgt mutant modifies the expression level of genes related to RG-I synthesis. 
Whether the changes in gene expression level observed for these genes compensate for the lack of URGTs will need to be further explored.

The expression of URGT2, URGT4, and URGT6 is finely regulated in seed coat epidermal cells

A network of transcription regulators has been described to control the differentiation of seed coat epidermal cells. Some of these have been implicated in the regulation of genes involved in the production of mucilage polysaccharides (Golz et al., 2018). Notably, GL2 is required for MUM4 and GATL5 expression, TTG2 and MYB61 also positively regulate GATL5 expression, and LUH/MUM1 controls the expression of $\beta$-GALACTOSIDASE6 (BGAL6/MUM2) and $\beta$-XYLOSIDASE1 (BXL1) genes that were previously reported to have an impact on RG-I maturation (Western et al., 2004; Dean et al., 2007; Macquet et al., 2007b; Huang et al., 2011; Walker et al., 2011; Kong et al., 2013). Similarly, the expression of URGT2 and URGT4 was reduced in developing seeds of $g / 2$, ttg2, mum1, and myb61 (Figure 10). In contrast, URGT6 appeared to be repressed only by MUM1 (Figure 10).

This difference in the regulation of URGT6 compared to URGT2 and URGT4 agrees with a more specific role in AM RG-I production compared to the stronger contribution of URGT2 and URGT4 to RG-I synthesis, as demonstrated by the extent of impaired polymer production in the urgt2urgt4 mutant. Figure 11 presents our proposed model for the regulation of URGT2, URGT4, URGT6 and additional mucilage specific UDP-Sugars transporters (UUAT1 and UUAT3) by different transcription factors acting on mucilage modification and synthesis (Figure 11 and Supplemental Figure 15). However, it is important to note that these factors are required for URGT2, URGT4, URGT6, UUAT1 and UUAT2 expression, and more experiments are required to demonstrate this proposed model.

RG-I production in seed coat mucilage is fine-tuned by URGT2, URGT4, and URGT6 regulating UDP-rhamnose availability in the Golgi apparatus 
Previous studies demonstrated that URGT2, URGT4, and URGT6 are proteins with the same function of importing UDP-Rha and UDP-Gal from the cytosol to the lumen of the Golgi apparatus (Rautengarten et al., 2014). However, even though they exhibit the same in vitro activity, their mutation results in different phenotypes; thus, these results suggest that the three transporters contribute in different ways to mucilage RG-I biosynthesis. URGT2 and URGT4 mainly affect the length of SM RG-I, whereas URGT6 and URGT4 also affect AM structuration. Changes in the xylose amount, which maintain the Xyl-RG-I ratio in the mutant similar as in the wild-type, confirm the importance of xylan domains in RG-I mucilage polymer production, suggesting the possible coordination of the enzymes responsible for addition and synthesis of the xylan chains and those synthesizing the RG-I backbone, as proposed in Fabrissin et al. (2019). How do URGT2, URGT4, and URGT6 contribute in different manners to mucilage biosynthesis and its structuration? A possible explanation is a formation in the Golgi of specific complexes with different combinations of GTs and URGTs, where each URGT plays a different role in supplying the building blocks for RG-I biosynthesis with different characteristics. The identification of glycosyltransferases and other proteins involved in RG-I biosynthesis should provide more tools to investigate this hypothesis.

\section{Material and Methods}

\section{Plant Material and Growth Conditions}

The urgt1-2 (SAIL_768_C08), urgt2-1 (SALK_125196), urgt2-2 (SALK_071647), urgt3-2 (SAIL_273_E01), urgt4-2 (SALK_152675), urgt4-3 (FLAG_424A06), urgt5-1 (SALK_059372C), urgt6-1 (GK-099E03) and urgt6-2 (SALK_061826) mutants were obtained from the ABRC (http://abrc.osu.edu/) using the SIGnAL Salk collection (Alonso et al., 2003) and from the Versailles Arabidopsis Stock Center (Jean-Pierre Bourgin Institute, INRA). Homozygous lines were selected by PCR using the primers indicated in Supplemental Table 4 on the genomic DNA of each mutant. Mutants in transcription regulators, gl2-6, myb61, mum1/luh-3, and ttg2-3 have been described previously 
(Ishida et al., 2007; Saez-Aguayo et al., 2013; 2014). Plant growth was performed in either a growth chamber (photoperiod of $16 \mathrm{~h}$ light at $21^{\circ} \mathrm{C}, 8 \mathrm{~h}$ dark at $18^{\circ} \mathrm{C}, 65 \%$ relative humidity, and $170 \mu \mathrm{mol} \mathrm{m} \mathrm{m}^{-2} \mathrm{~s}^{-1}$ ) or a greenhouse $\left(18\right.$ to $\left.28^{\circ} \mathrm{C}\right)$ with a minimum photoperiod of $13 \mathrm{~h}$ assured by supplemental lighting. Plants were grown in compost (Stender substrate for Versailles and Top Crop substrate for Santiago) and watered with Plan-Prod nutritive solution (Fertil for Versailles). In all comparative analyses, seeds used were from a pool of approximately 10 mutant and wild-type plants that had been simultaneously cultivated and harvested from at least 3 biological repeats.

\section{Cloning procedure of urgt2-2, urgt4-2 and urgt6-1 molecular rescue}

The entire coding sequence of URGT2, URGT4, and URGT6 were cloned from cDNA synthesized from Arabidopsis leaf RNA. All sequences without native stop codon were PCR-amplified using the following primer pairs: FwURGT2: 5'-CACCATGGAGAAAGCAGAGAACGAGA-3', RevURGT2: 5'-TGCTTTATTATTTCCAAGCTCCAT-3', FwURGT4: 5'-CACCATGTTTAAAAAAATGAGTGCT-3', RevURGT4: 5'-CACCTTTTCATCAGATTCGTTTGT-3', FWURGT6: 5'-CACCATGGCTCCAGTGAGTAAAGCTG-3', RevURGT6: 5'-GGCTTTGTCTTCGTTGTCGTC -3'. Resulting PCR products were introduced into the pENTR $^{\mathrm{TM}} / \mathrm{D}-\mathrm{TOPO}{ }^{\circledR}$ cloning vector according to the standard protocol (Thermo Fisher Scientific) to generate the entry clones pENTR-URGT2, pENTR-URGT4, and pENTR-URGT6. The intergenic region of 778bp between At1g21080 and At1g21070 genes, 2038bp between At4g39390 and At4g39380 genes and 2012bp between At1g34020 and At1g34010 genes was defined as the URGT2 (proURGT2), URGT4 (proURGT4) and URGT6 (proURGT6) promoters, respectively. Those promoter regions were amplified by PCR from Arabidopsis genomic DNA using the following primers: proURGT2Fw: 5'-TCATGTGTTGCGAATCTTATTC-3', proURGT2Rev: 5'-TTGGATTCAAATTAAAAAAATTCGAAATCTGAAATC-3' proURGT4Fw: 5'ATACGAGTCAGTAGATGTGCA-3', proURGT4Rev: 5'-CTCAAATTCAGATCTCTGCA-3', proURGT6: 5'-AGTCATATCATTAATAAAA-3', proURGT6Rev 5'-TTTTCTGAACTAACTTTCTCT-3'. PCR products were introduced 
into the pENTRTM/ 5'-TOPO ${ }^{\circledR}$ vector (Thermo Fisher Scientific) to generate the pENTR5-pURGT2, pENTR5-pURGT4, and pENTR5-pURGT6 entry clones. C-terminal GFP fusion was obtained by recombination of the entry clones for promoter and CDS regions of each gene with the destination vector R4pGWB504 (Nakagawa T. et al., 2008), using LR clonase (Thermo Fisher Scientific). The resulting constructs were introduced into the respective $A$. thaliana urgt2-2, urgt4-2, and urgt6-1 mutant lines through the Floral Dip method (Clough and Bent, 1998). At least three resistant lines for each genetic rescue were selected.

\section{Expression Analysis}

Different tissue organs were collected at indicated days of growth, and seeds were dissected from approximately four siliques in each Days After Pollination (DAP) $(6,8$, 10 , and 12 DAP) for further RNA extraction. RNA was extracted following the procedure of RNeasy Plus Micro Kit (Qiagen). One microgram of total RNA, with a previous DNAse I (Invitrogen) treatment, was used as a template for first-strand cDNA synthesis with oligo(dT) primer and SuperScript II (Thermo Fisher Scientific), according to the manufacturer's instructions. Measurements were realized from at least 3 different plants. For RT-PCR analysis, primers described in Supplemental Table S4 were used to amplify the entire coding sequence of URGTs from single-stranded cDNA in the wild-type Col-0, urgt2-2, urgt4-2, urgt6-1, double and triple mutants. The primers used to amplify EF1aA4 were those described by North et al. (2007). RT-qPCR was performed using the Fast EvaGreen qPCR Master Mix kit (Mx3000P; Stratagene). Reactions containing $1 \mu \mathrm{L}$ of $1: 2$ diluted cDNA in a total volume of $10 \mu \mathrm{L}$ were performed using primers that have been previously tested for their efficiency rates and sensitivity in a cDNA dilution series. The quantification and normalization procedures were done using the equation described in Saez-Aguayo et al., 2017. EF1aA4 (At5g60390), Clathrin adaptor (At5g46630), and the seed reference gene At4g12590 (Hong et al., 2010) were used as reference genes, and all primers used in this study are described in Supplemental Table S4.

\section{Histological techniques}


For FITC-dextran staining, dry seeds were incubated in $300 \mu \mathrm{L}$ of $1 \mathrm{mg} \mathrm{mL}-1$ FITC-dextran 70-kD molecules (Sigma-Aldrich; 46945) for $30 \mathrm{~min}$ at $125 \mathrm{rpm}$. Seeds were transfered to glass slides and imaged with a Leica TCS SP8 confocal system A 488-nm neon laser line was used to excite the molecule. The laser intensity was fixed at $5 \%$, collection bandwidth fixed between $502-542 \mathrm{~nm}$, and the gain at 900 . Image $\mathrm{J} 1.52 \mathrm{a}$ Software (Schindelin et al., 2015), was used to analyze and quantify the FITC-dextran 70-kD penetration and the yellow/orange pixel quantification contained in the inner adherent mucilage. Images were acquired by generating black and white 8-bit image with a grayscale of pixel intensity values from 0 to 255, where 0 correspond to the absence and 255 to the total presence of FITC-dextran 70-kD. A set threshold was used to separate seed (without mucilage) and the mucilage outline between the FITC-dextran colored and not colored zones. Seed diameters and mucilage area were recorded to obtain the mucilage width and to quantify the pixel intensity observed in the inner adherent mucilage following the equation:

Where "IntMuci" and "AreaMuci1" are the measures of pixel intensity and area, respectively, of the mucilage pocket. In the same way, "IntSeed" and "AreaSeed" are the measures within the circumference that encloses the seed without considering the mucilage, and IntBackground is an average of pixel intensity measurements inside the seed used as a background.

For mature seed size determination, FITC-dextran staining images were used. For WT Col-0 and triple urgt mutant line, 65 seed images from 3 biological replicates were used. Image J v.1.52a software (Schindelin et al., 2015) threshold was adjusted to delimit and mark the area of the seeds without considering mucilage, from which the total area of the seed was determined. Student's t-test with $p<0,05$ was performed to determine 
significant differences.

\section{Extraction and analysis of mucilage layers and demucilaged seeds}

For a quick screening of the urgt single mutants impact on the soluble mucilage composition, $50 \mathrm{mg}$ of dry seeds were imbibed three times with $2 \mathrm{~mL}$ of water for 20 min. Supernatants were collected after $10 \mathrm{~min}$ of centrifugation at $8000 \mathrm{~g}$ and mixed to form the SM fraction. The SM was later lyophilized, resuspended in $600 \mu \mathrm{L}$ of deionized water and stored at $-20^{\circ} \mathrm{C}$ until monosaccharide analysis.

To gain a deeper knowledge of the changes in mucilage composition and structuration of the urgt single, double and triple mutants, a sequential extraction procedure was used to obtain the soluble mucilage layer, the adherent mucilage layer and demucilaged seeds. The soluble mucilage was extracted from $200 \mathrm{mg}$ of dry seeds by mixing for $3 \mathrm{~h}$ in $4 \mathrm{~mL}$ of distilled water at RT. Supernatants were recovered after centrifugation at $8,000 \mathrm{~g}$ for $10 \mathrm{~min}$, filtered through a disposable glass microfiber filter (13 $\mathrm{mm}$ diameter, $2.7 \mathrm{~mm}$ pore size; Whatman) and stored at $-20^{\circ} \mathrm{C}$ before sugar and macromolecular analysis. After soluble mucilage extraction, seeds+AM were rinsed twice with $4 \mathrm{~mL}$ of distilled water. For digestion and separation of the adherent layer, hydrated seeds+AM

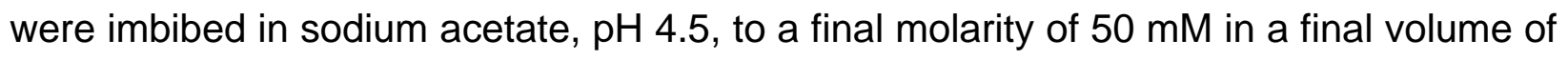
$4 \mathrm{~mL}$. Rhamnogalacturonan hydrolase (Swiss-Prot Q00018) provided by Novozymes was added ( 0.1 nkat), and samples were incubated overnight at $40^{\circ} \mathrm{C}$ to digest the $\mathrm{AM}$ and separate them from the seed surface. After centrifugation $(8,000 \mathrm{~g}, 3 \mathrm{~min})$, supernatants containing the depolymerized $\mathrm{AM}$ were collected and stored at $-20^{\circ} \mathrm{C}$ for sugar analysis. The demucilaged seeds were rinsed three times with distilled water, freeze-dried stored at $-20^{\circ} \mathrm{C}$ until their analysis.

\section{AIR Preparation, acid hydrolysis and sugar analysis}

Freeze-dried demucilaged seeds were ground under liquid nitrogen and lipids were removed by incubating overnight with methanol:chloroform $(2: 1, v: v)$ followed by two washes of $2 \mathrm{~h}$ with the same solution. The residue was collected by centrifugation and the defatted and demucilaged seed fraction was dried overnight at room temperature 
followed by $2 \mathrm{~h}$ in a vacuum oven.

For a quick screening of urgt single mutants, SM was hydrolyzed with $2 \mathrm{M}$ trifluoroacetic acid (TFA) at $121^{\circ} \mathrm{C}$ for $1 \mathrm{~h} 30 \mathrm{~min}$, followed by termination of the hydrolysis by cooling the samples. TFA was evaporated at $60^{\circ} \mathrm{C}$ under nitrogen gas and the samples were washed twice with $400 \mathrm{~mL}$ of $100 \%$ isopropanol and dried with nitrogen. Hydrolyzed products were resuspended with water and filtered by passing through a syringe filter (pore size: $0.45 \mathrm{~mm}$ ), transferred to a new tube, and used for HPAEC-PAD (Dionex ICS3000) analysis as described Saez-Aguayo et al., 2017; 0,25 mM of Myo-inositol and allose were used as internal standards.

For a deeper analysis of the changes in the mucilage composition of the urgt single, double, and triple mutants, we quantified the individual neutral sugars and the uronic acid sugars on SM, AM, and deffated demucilaged seeds. Uronic acid quantification (considered as GalA) was determined by the automated m-hydroxybiphenyl method (Thibault, 1979). The individual neutral sugars analysis was performed by the quantification of their alditol acetate derivatives (Blakeney et al., 1983) by gas-liquid chromatography after hydrolysis with $2 \mathrm{M}$ trifluoroacetic acid at $121^{\circ} \mathrm{C}$ for $2.5 \mathrm{~h}$ for soluble fractions (SM and $\mathrm{AM}$ ) and prehydrolysis (26 N H2SO4, $1 \mathrm{~h}, 25^{\circ} \mathrm{C}$ ), followed by hydrolysis $\left(2 \mathrm{~N} \mathrm{H} 2 \mathrm{SO} 4,2 \mathrm{~h}, 100^{\circ} \mathrm{C}\right.$ ) for insoluble fractions (deffated demucilaged seeds). The adherent mucilage was extracted using rhamnogalacturonan hydrolase, an enzyme that is not expected to cause substantial cellular disruption in contrast to other methods (Zhao et al., 2017). Therefore, we assumed that starch contamination would be minimal; thus, we did not treat the samples with amylase.

\section{HP-SEC-MALLS-Viscometer analysis of soluble mucilage layer}

For analysis of the macromolecular characteristics of soluble mucilage, $1 \mathrm{~mL}$ of SM was vigorously shaken, boiled for $5 \mathrm{~min}$, and filtered through a polyvinylidene difluoride filter (13 $\mathrm{mm}$ diameter, $0.45 \mathrm{~mm}$ pore size; Whatman) prior to HP-SEC injection. HP-SEC analysis was performed at room temperature on a system comprising a Shodex $\mathrm{OH}$ SB-G precolumn followed by a Shodex OH-Pack SB-805 HQ column 
(http://www.shodex.de/). Elution was carried out with $50 \mathrm{mM}$ sodium nitrate buffer at a constant flow rate of $60 \mathrm{~mL} \cdot \mathrm{h}^{-1}$. Measurements were performed using a differential refractometer (VE 3580 RI Detector) and a Viscotek 270 Dual Detector (dual laser light scattering, I = $670 \mathrm{~nm}, 90$ and 7, combined with a differential pressure viscometer; Malvern Instruments; http://www.malvern.com/). All detectors were calibrated with a pullulan standard that has narrow molecular mass distribution $(\mathrm{Mw}=145,618 \mathrm{Da}, \mathrm{Mn}=$ $139,180 \mathrm{Da},[\mathrm{n}]=54.0 \mathrm{~mL} \mathrm{~g}^{-1}$ at $30 \mathrm{C}$ in $0.1 \mathrm{M}$ sodium nitrate, $\mathrm{dn} / \mathrm{dc}=0.147 \mathrm{~mL} \cdot \mathrm{g}^{-1}$; Malvern Instruments). Samples were automatically injected through a $50 \mu \mathrm{L}$ loop. Data analyses were performed using OmniSec version 4.5 software (Malvern Instruments).

Calculation of the number of RG-I molecules was carried out using the following equation as published in Fabrissin et al., 2019:

Where corresponds to SM Rha + GalA content expressed in moles; is the Avogadro constant $\left(6.022 \times 10^{23} \mathrm{~mol}^{-1}\right)$; and refers to the number of $\{$ Rha-GalA $\}$ dimers calculated for the RG-I molecule for each genotype (Supplemental Table 2).

\section{Linkage analysis of the soluble mucilage fraction}

Soluble mucilage samples were solubilized in water with a final sugar concentration between 0.5 and $1 \mathrm{~g}$ of sugars $/ \mathrm{mL}$ and converted into their $\mathrm{H}^{+}$form by percolating the solutions through a $1 \mathrm{~mL}$ Sigma Dowex 50WX4 resin. Samples were freeze-dried and further dried in a vacuum oven at $40^{\circ} \mathrm{C}$ for $2 \mathrm{~h}$ before being dissolved in $0.2 \mathrm{~mL}$ of dimethyl sulfoxide. Mixtures were left for $30 \mathrm{~min}$ at room temperature with occasional vortexing before adding $0.2 \mathrm{~mL}$ of $\mathrm{NaOH}$-dimethyl sulfoxide solution. Methylation was performed with $0.1 \mathrm{~mL}$ of methyl iodide for $10 \mathrm{~min}$. The mixture was sonicated and vortexed several times. Two milliliters of water were added, and methylated products were extracted with $2 \mathrm{~mL}$ of chloroform. The organic phase was washed three times with $2 \mathrm{~mL}$ of water and dried $\left(45^{\circ} \mathrm{C}\right)$. Methylated carbohydrates were hydrolyzed with $2 \mathrm{M}$ trifluoroacetic acid and converted to their alditol acetates. The partially methylated alditol acetates were analyzed by gas chromatography-mass spectrometry. 


\section{Accession Numbers}

Sequence data from this article can be found in the GenBank/EMBL data libraries under accession numbers KP872774 (URGT2), KJ667160.1 (URGT4), and KJ667162.1 (URGT6).

\section{Acknowledgments}

(To S.S-A), 1151335 (to A.O), 3170796 (to A.L.G), Fondo de Areas Prioritarias- Centro de Regulacion del Genoma-15090007, ECOSud-CONICYT C14B02 (to A.O., H.M.N and M.-C.R) and PAI 79170136 to (S.S-A). We thank Miriam Barros for her advice and expertise in confocal microscopy. We also thanks Marie-Jeanne Crépeau and Alvaro Miquel for technical assistance with HPSEC, GC (To M-JC) and HPAEC analysis (To AM).

\section{Supplemental Data}

\section{Supplemental Figure Legends}

Supplemental Figure 1: URGT gene expression in planta

Supplemental Figure 2: URGT gene expression profiles in developing seed tissues

Supplemental Figure 3: Arabidopsis seedling morphology of urgt2-2, urgt4-2 and urgt6-1 in comparison to the wild-type

Supplemental Figure 4: Characterization of urgt2, urgt4, and urgt6 mutant lines

Supplemental Figure 5: Quantification of URGT4 and URGT6 expression in urgt4 and urgt6 mutant lines

Supplemental Figure 6: Immmunolabelling of rhamnogalacturonan-I in the adherent mucilage of urgt2, urgt4, and urgt6 mutant alleles

Supplemental Figure 7: Molecular complementation of adherent mucilage 
rhamnogalacturonan-I labeling in the urgt2 mutant

Supplemental Figure 8: Molecular complementation of adherent mucilage rhamnogalacturonan-I labeling in the urgt4 mutant

Supplemental Figure 9: Molecular complementation of adherent mucilage rhamnogalacturonan-I labeling in the urgt6 mutant

Supplemental Figure 10: URGT expression in different urgt single, double, and triple mutant combinations

Supplemental Figure 11: Quantification of URGT1, URGT3, and URGT5 expression in urgt single, double, and triple mutants

Supplemental Figure 12: Size of mature dry seeds from urgt mutant line

Supplemental Figure 13: GL2, TTG2, MUM1, and MYB61 expression in developing wild-type seeds

Supplemental Figure 14 RG-I length and molecule number contents of the soluble mucilage layer are tightly proportional except in urgt mutant lines

Supplemental Figure 15: Modulation of UUAT1 and UUAT3 expression by MUM1, MYB61, TTG1 and GL2 transcription regulators

\section{Supplemental Tables}

Supplemental Table 1: Sugar content in the soluble mucilage layer of the URGT mutant lines

Supplemental Table 2: Mucilage phenotypes of urgt2, urgt4, and urgt6 mutant lines Supplemental Table 3: RG-I length in mucilage of the urgt mutant lines Supplemental Table 4: Primers used for this study

\section{Tables}

Table 1: Monosaccharide composition of adherent and soluble mucilage layers and demucilaged seeds of the urgt mutant lines compared to the wild-type.

Mucilage was sequentially extracted with water followed by enzymatic digestion with rhamnogalacturonan hydrolase, and sugar contents determined for soluble and adherent mucilage or demucilaged seeds. Values represent the means and SD (in 
parentheses) of 3-4 biological repeats $(n=3-4)$. AM, adherent mucilage; SM, soluble mucilage; DS, demucilaged seed. Letters represents differences between different genotypes with statistical analyses performed using the Mann and Whitney test with $p<0.05$.

\begin{tabular}{|c|c|c|c|c|c|c|c|c|}
\hline \multicolumn{9}{|c|}{ Soluble mucilage polysaccharides ( $\mathrm{mg} / \mathrm{g}$ of dry seed) } \\
\hline Sugars & $\begin{array}{c}\text { WT } \\
\text { Col-0 }\end{array}$ & urgt2-2 & urgt4-2 & urgt6-1 & $\begin{array}{l}\text { urgt2-2 } \\
\text { urgt4-2 }\end{array}$ & $\begin{array}{l}\text { urgt2-2 } \\
\text { urgt6-1 }\end{array}$ & $\begin{array}{l}\text { urgt4-2 } \\
\text { urgt6-1 }\end{array}$ & $\begin{array}{c}\text { Triple } \\
\text { urgt }\end{array}$ \\
\hline GalA & $\begin{array}{c}9.29 \\
(0.30) \mathrm{a}\end{array}$ & $\begin{array}{c}7.03 \\
(0.47) \mathrm{b}\end{array}$ & $\begin{array}{c}9.61 \\
(0.40) \mathrm{a}\end{array}$ & $\begin{array}{c}9.63 \\
(0.45) \mathrm{a}\end{array}$ & $\begin{array}{c}6.31 \\
(0.38) \mathrm{b}\end{array}$ & $\begin{array}{c}8.19 \\
(0.36) \mathrm{c}\end{array}$ & $\begin{array}{c}8.62 \\
(0.23) \mathrm{c}\end{array}$ & $\begin{array}{c}5.80 \\
(0.53) d\end{array}$ \\
\hline Rha & $\begin{array}{l}11.08 \\
(0.7) \mathrm{a}\end{array}$ & $\begin{array}{c}9.33 \\
(0.6) \mathrm{b}\end{array}$ & $\begin{array}{c}9.47 \\
(1.0) \mathrm{b}\end{array}$ & $\begin{array}{l}10.22 \\
(1.7) \mathrm{a}\end{array}$ & $\begin{array}{c}6.09 \\
(0.1) \mathrm{d}\end{array}$ & $\begin{array}{c}8.06 \\
(0.5) \mathrm{c}\end{array}$ & $\begin{array}{c}9.00 \\
(0.1) \mathrm{b}\end{array}$ & $\begin{array}{c}6.71 \\
(0.7) \mathrm{d}\end{array}$ \\
\hline Ara & $\begin{array}{c}0.15 \\
(0.07)\end{array}$ & $\begin{array}{c}0.10 \\
(0.004)\end{array}$ & $\begin{array}{c}0.11 \\
(0.02)\end{array}$ & $\begin{array}{c}0.13 \\
(0.03)\end{array}$ & $\begin{array}{c}0.10 \\
(0.01)\end{array}$ & $\begin{array}{c}0.13 \\
(0.02)\end{array}$ & $\begin{array}{c}0.11 \\
(0.003)\end{array}$ & $\begin{array}{c}0.13 \\
(0.02)\end{array}$ \\
\hline Xyl & $\begin{array}{c}0.59 \\
(0.08) \mathrm{a}\end{array}$ & $\begin{array}{c}0.66 \\
(0.08) a\end{array}$ & $\begin{array}{c}0.61 \\
(0.03) \mathrm{a}\end{array}$ & $\begin{array}{c}0.56 \\
(0.04) \mathrm{a}\end{array}$ & $\begin{array}{c}0.89 \\
(0.04) \mathrm{b}\end{array}$ & $\begin{array}{c}0.75 \\
(0.01) \mathrm{c}\end{array}$ & $\begin{array}{c}0.73 \\
(0.02) \mathrm{c}\end{array}$ & $\begin{array}{c}1.36 \\
(0.22) d\end{array}$ \\
\hline Man & $\begin{array}{c}0.25 \\
(0.09) \mathrm{a}\end{array}$ & $\begin{array}{c}0.17 \\
(0.02) \mathrm{b}\end{array}$ & $\begin{array}{c}0.25 \\
(0.02) a\end{array}$ & $\begin{array}{c}0.20 \\
(0.05) \mathrm{a}\end{array}$ & $\begin{array}{c}0.12 \\
(0.02) \mathrm{c}\end{array}$ & $\begin{array}{c}0.15 \\
(0.05) \mathrm{b}\end{array}$ & $\begin{array}{c}0.17 \\
(0.05) \mathrm{b}\end{array}$ & $\begin{array}{c}0.24 \\
(0.02) \mathrm{a}\end{array}$ \\
\hline Gal & $\begin{array}{c}0.41 \\
(0.15) \mathrm{a}\end{array}$ & $\begin{array}{c}0.24 \\
(0.03) \mathrm{b}\end{array}$ & $\begin{array}{c}0.35 \\
(0.03) \mathrm{a}\end{array}$ & $\begin{array}{c}0.22 \\
(0.03) \mathrm{b}\end{array}$ & $\begin{array}{c}0.20 \\
(0.01) \mathrm{b}\end{array}$ & $\begin{array}{c}0.33 \\
(0.01) \mathrm{a}\end{array}$ & $\begin{array}{c}0.26 \\
(0.02) \mathrm{b}\end{array}$ & $\begin{array}{c}0.25 \\
(0.07) \mathrm{b}\end{array}$ \\
\hline Glc & $\begin{array}{c}0.30 \\
(0.15) \mathrm{a}\end{array}$ & $\begin{array}{c}0.29 \\
(0.07) \mathrm{a}\end{array}$ & $\begin{array}{c}0.41 \\
(0.06) \mathrm{a}\end{array}$ & $\begin{array}{c}0.33 \\
(0.07) \mathrm{a}\end{array}$ & $\begin{array}{c}0.18 \\
(0.05) \mathrm{b}\end{array}$ & $\begin{array}{c}0.30 \\
(0.05) a\end{array}$ & $\begin{array}{c}0.22 \\
(0.11) a\end{array}$ & $\begin{array}{c}0.29 \\
(0.02) \mathrm{a}\end{array}$ \\
\hline $\begin{array}{l}\text { Total sugars } \\
\text { (SM) }\end{array}$ & $\begin{array}{l}22.06 \\
(0.49) \mathrm{a}\end{array}$ & $\begin{array}{c}17.83 \\
(0.93) \mathrm{C}\end{array}$ & $\begin{array}{c}20.81 \\
(0.52) \mathrm{b}\end{array}$ & $\begin{array}{l}21.30 \\
(1.49) \mathrm{a}\end{array}$ & $\begin{array}{l}13.89 \\
(0.30) d\end{array}$ & $\begin{array}{c}17.90 \\
(0.10) \mathrm{c}\end{array}$ & $\begin{array}{c}19.10 \\
(0.26) \mathrm{c}\end{array}$ & $\begin{array}{c}14.78 \\
(0.95) d\end{array}$ \\
\hline \multicolumn{9}{|c|}{ Adherent mucilage polysaccharides (mg/g of dry seed) } \\
\hline GalA & $\begin{array}{c}5.62 \\
(0.04) \mathrm{a}\end{array}$ & $\begin{array}{c}4.84 \\
(0.24) \mathrm{b}\end{array}$ & $\begin{array}{c}5.75 \\
(0.37) \mathrm{a}\end{array}$ & $\begin{array}{c}5.93 \\
(0.42) \mathrm{a}\end{array}$ & $\begin{array}{c}3.49 \\
(0.08) \mathrm{c}\end{array}$ & $\begin{array}{c}4.75 \\
(0.13) \mathrm{b}\end{array}$ & $\begin{array}{c}5.76 \\
(0.06) \mathrm{a}\end{array}$ & $\begin{array}{c}3.57 \\
(0.09) c\end{array}$ \\
\hline Rha & $\begin{array}{c}4.14 \\
(0.19) \mathrm{a}\end{array}$ & $\begin{array}{l}3.53 \\
(0.19) c\end{array}$ & $\begin{array}{c}4.0 \\
(0.21) \mathrm{a}\end{array}$ & $\begin{array}{c}3.68 \\
(0.07) \mathrm{b}\end{array}$ & $\begin{array}{c}2.85 \\
(0.22) d\end{array}$ & $\begin{array}{c}3.40 \\
(0.20) c\end{array}$ & $\begin{array}{c}3.81 \\
(0.08) b\end{array}$ & $\begin{array}{c}2.46 \\
(0.18) d\end{array}$ \\
\hline Ara & $\begin{array}{c}0.25 \\
(0.01) \mathrm{a}\end{array}$ & $\begin{array}{c}0.26 \\
(0.03) \mathrm{a}\end{array}$ & $\begin{array}{c}0.26 \\
(0.01) \mathrm{a}\end{array}$ & $\begin{array}{c}0.26 \\
(0.01) \mathrm{a}\end{array}$ & $\begin{array}{c}0.22 \\
(0.01) \mathrm{b}\end{array}$ & $\begin{array}{c}0.18 \\
(0.03) \mathrm{b}\end{array}$ & $\begin{array}{c}0.23 \\
(0.003) \mathrm{b}\end{array}$ & $\begin{array}{c}0.25 \\
(0.003) a\end{array}$ \\
\hline Xyl & $\begin{array}{c}0.14 \\
(0.01) \mathrm{a}\end{array}$ & $\begin{array}{c}0.11 \\
(0.02) \mathrm{b}\end{array}$ & $\begin{array}{c}0.11 \\
(0.03) \mathrm{b}\end{array}$ & $\begin{array}{c}0.12 \\
(0.03) \mathrm{b}\end{array}$ & $\begin{array}{c}0.10 \\
(0.02) \mathrm{b}\end{array}$ & $\begin{array}{c}0.09 \\
(0.03) \mathrm{c}\end{array}$ & $\begin{array}{c}0.09 \\
(0.02) \mathrm{c}\end{array}$ & $\begin{array}{c}0.11 \\
(0.04) \mathrm{b}\end{array}$ \\
\hline Man & $\begin{array}{c}0.29 \\
(0.02)\end{array}$ & $\begin{array}{c}0.28 \\
(0.02)\end{array}$ & $\begin{array}{c}0.25 \\
(0.03)\end{array}$ & $\begin{array}{c}0.28 \\
(0.02)\end{array}$ & $\begin{array}{c}0.27 \\
(0.01)\end{array}$ & $\begin{array}{c}0.27 \\
(0.08)\end{array}$ & $\begin{array}{c}0.28 \\
(0.03)\end{array}$ & $\begin{array}{c}0.25 \\
(0.01)\end{array}$ \\
\hline Gal & $\begin{array}{c}2.30 \\
(0.10) a\end{array}$ & $\begin{array}{c}2.56 \\
(0.13) \mathrm{b}\end{array}$ & $\begin{array}{c}2.22 \\
(0.13) \mathrm{a}\end{array}$ & $\begin{array}{c}2.33 \\
(0.07) \mathrm{a}\end{array}$ & $\begin{array}{c}2.21 \\
(0.07) \mathrm{a}\end{array}$ & $\begin{array}{c}2.30 \\
(0.21) a\end{array}$ & $\begin{array}{c}2.24 \\
(0.10) a\end{array}$ & $\begin{array}{c}2.42 \\
(0.02) \mathrm{c}\end{array}$ \\
\hline Glc & $\begin{array}{c}11.72 \\
(1.03) \mathrm{a}\end{array}$ & $\begin{array}{c}10.69 \\
(0.33) a\end{array}$ & $\begin{array}{c}11.17 \\
(0.78) a\end{array}$ & $\begin{array}{c}11.45 \\
(0.67) \mathrm{a}\end{array}$ & $\begin{array}{c}13.09 \\
(0.94) \mathrm{b}\end{array}$ & $\begin{array}{c}11.16 \\
(1.91) a\end{array}$ & $\begin{array}{c}10.33 \\
(0.50) a\end{array}$ & $\begin{array}{c}11.58 \\
(0.64) a\end{array}$ \\
\hline $\begin{array}{l}\text { Total sugars } \\
\text { (AM) }\end{array}$ & $\begin{array}{l}24.45 \\
(0.91) \mathrm{a}\end{array}$ & $\begin{array}{l}22.27 \\
(0.54) \mathrm{b}\end{array}$ & $\begin{array}{l}23.74 \\
(0.95) \mathrm{a}\end{array}$ & $\begin{array}{l}24.06 \\
(0.79) \mathrm{a}\end{array}$ & $\begin{array}{l}22.21 \\
(0.78) \mathrm{b}\end{array}$ & $\begin{array}{l}22.17 \\
(1.77) \mathrm{b}\end{array}$ & $\begin{array}{l}22.75 \\
(0.25) \mathrm{b}\end{array}$ & $\begin{array}{l}20.66 \\
(0.48) c\end{array}$ \\
\hline
\end{tabular}




\begin{tabular}{|c|c|c|c|c|c|c|c|c|}
\hline $\begin{array}{l}\text { Total sugars } \\
\text { (SM+AM) }\end{array}$ & $\begin{array}{c}46.51 \\
(1.40) a \\
\end{array}$ & $\begin{array}{c}40.10 \\
(0.62) b \\
86 ?\end{array}$ & $\begin{array}{c}44.55 \\
(0.96) \mathrm{a} \\
058\end{array}$ & $\begin{array}{c}45.35 \\
(2.11) a \\
075\end{array}$ & $\begin{array}{c}36.10 \\
(1.08) d\end{array}$ & $\begin{array}{c}40.07 \\
(1.80) \mathrm{b}\end{array}$ & $\begin{array}{c}41.85 \\
(0.36) \mathrm{C} \\
909\end{array}$ & $\begin{array}{c}35.44 \\
(1.42) d \\
76 ?\end{array}$ \\
\hline $\begin{array}{l}\text { Total sugars } \\
(\mathrm{SM}+\mathrm{AM}) \quad(\%)\end{array}$ & $\begin{array}{l}100.0 \\
(3.0)\end{array}$ & $\begin{array}{l}86.2 \\
(1.3)\end{array}$ & $\begin{array}{l}95.8 \\
(2.1)\end{array}$ & $\begin{array}{l}97.5 \\
(4.5)\end{array}$ & $\begin{array}{l}77.6 \\
(2.3)\end{array}$ & $\begin{array}{l}86.1 \\
(3.9)\end{array}$ & $\begin{array}{l}90.9 \\
(0.8)\end{array}$ & $\begin{array}{l}76.2 \\
(3.1)\end{array}$ \\
\hline \multicolumn{9}{|c|}{ Demucilaged seed polysaccharides ( $\mathrm{mg} / \mathrm{g}$ of dry seed) } \\
\hline GalA & $\begin{array}{l}26.04 \\
(2.79)\end{array}$ & $\begin{array}{l}27.49 \\
(2.00)\end{array}$ & $\begin{array}{l}27.87 \\
(2.16)\end{array}$ & $\begin{array}{l}26.91 \\
(1.79)\end{array}$ & $\begin{array}{l}26.91 \\
(3.16)\end{array}$ & $\begin{array}{l}27.09 \\
(1.49)\end{array}$ & $\begin{array}{l}26.32 \\
(4.23)\end{array}$ & $\begin{array}{l}26.64 \\
(1.96)\end{array}$ \\
\hline Rha & $\begin{array}{l}5.03 \\
(0.56)\end{array}$ & $\begin{array}{c}4.97 \\
(0.32)\end{array}$ & $\begin{array}{c}5.07 \\
(0.53)\end{array}$ & $\begin{array}{c}4.63 \\
(0.63)\end{array}$ & $\begin{array}{c}4.46 \\
(0.33)\end{array}$ & $\begin{array}{c}5.40 \\
(1.63)\end{array}$ & $\begin{array}{c}4.27 \\
(0.49)\end{array}$ & $\begin{array}{c}4.56 \\
(0.69)\end{array}$ \\
\hline Fuc & $\begin{array}{c}0.77 \\
(0.09)\end{array}$ & $\begin{array}{c}0.82 \\
(0.08)\end{array}$ & $\begin{array}{c}0.80 \\
(0.03)\end{array}$ & $\begin{array}{c}0.89 \\
(0.08)\end{array}$ & $\begin{array}{c}0.81 \\
(0.04)\end{array}$ & $\begin{array}{c}0.87 \\
(0.18)\end{array}$ & $\begin{array}{c}0.76 \\
(0.08)\end{array}$ & $\begin{array}{l}0.80 \\
(0.07)\end{array}$ \\
\hline Ara & $\begin{array}{l}23.03 \\
(1.99)\end{array}$ & $\begin{array}{l}22.64 \\
(1.93)\end{array}$ & $\begin{array}{l}24.08 \\
(2.07)\end{array}$ & $\begin{array}{l}23.66 \\
(1.51)\end{array}$ & $\begin{array}{l}24.36 \\
(0.67)\end{array}$ & $\begin{array}{l}24.40 \\
(4.31)\end{array}$ & $\begin{array}{l}22.65 \\
(1.70)\end{array}$ & $\begin{array}{l}22.80 \\
(1.37)\end{array}$ \\
\hline Xyl & $\begin{array}{c}7.72 \\
(0.76)\end{array}$ & $\begin{array}{l}8.01 \\
(0.58)\end{array}$ & $\begin{array}{l}8.24 \\
(0.48)\end{array}$ & $\begin{array}{l}8.05 \\
(0.78)\end{array}$ & $\begin{array}{l}8.18 \\
(0.15)\end{array}$ & $\begin{array}{c}8.26 \\
(1.62)\end{array}$ & $\begin{array}{c}7.87 \\
(0.60)\end{array}$ & $\begin{array}{l}8.01 \\
(0.42)\end{array}$ \\
\hline Man & $\begin{array}{l}2.94 \\
(0.32)\end{array}$ & $\begin{array}{l}2.77 \\
(0.31)\end{array}$ & $\begin{array}{l}3.13 \\
(0.41)\end{array}$ & $\begin{array}{l}2.88 \\
(0.16)\end{array}$ & $\begin{array}{l}3.04 \\
(0.26)\end{array}$ & $\begin{array}{l}3.14 \\
(0.83)\end{array}$ & $\begin{array}{l}2.84 \\
(0.27)\end{array}$ & $\begin{array}{l}3.13 \\
(0.53)\end{array}$ \\
\hline Gal & $\begin{array}{l}15.31 \\
(1.79)\end{array}$ & $\begin{array}{l}15.40 \\
(1.88)\end{array}$ & $\begin{array}{l}15.98 \\
(1.07)\end{array}$ & $\begin{array}{l}14.70 \\
(1.17)\end{array}$ & $\begin{array}{l}14.91 \\
(0.90)\end{array}$ & $\begin{array}{l}16.54 \\
(4.08)\end{array}$ & $\begin{array}{l}14.38 \\
(1.67)\end{array}$ & $\begin{array}{l}14.57 \\
(1.66)\end{array}$ \\
\hline Glc & $\begin{array}{l}38.24 \\
(3.87)\end{array}$ & $\begin{array}{l}37.76 \\
(3.94)\end{array}$ & $\begin{array}{l}38.83 \\
(1.68)\end{array}$ & $\begin{array}{l}36.57 \\
(4.15)\end{array}$ & $\begin{array}{l}38.96 \\
(1.46)\end{array}$ & $\begin{array}{l}39.40 \\
(10.77)\end{array}$ & $\begin{array}{l}35.89 \\
(3.17)\end{array}$ & $\begin{array}{l}38.79 \\
(5.83)\end{array}$ \\
\hline $\begin{array}{l}\text { Total sugars } \\
\text { (DS) }\end{array}$ & $\begin{array}{l}119.06 \\
(5.17)\end{array}$ & $\begin{array}{l}118.91 \\
(3.22)\end{array}$ & $\begin{array}{l}124.00 \\
(2.88)\end{array}$ & $\begin{array}{l}117.12 \\
(8.09)\end{array}$ & $\begin{array}{c}126.44 \\
(5.04)\end{array}$ & $\begin{array}{l}125.11 \\
(24.25)\end{array}$ & $\begin{array}{l}115.38 \\
(4.49)\end{array}$ & $\begin{array}{l}119.81 \\
(9.28)\end{array}$ \\
\hline
\end{tabular}

Table 2: Linkage analysis of tailored seed mucilage extracted from the triple urgt mutant line compared with the wild-type.

Values (average mol \% of neutral sugars) are shown from 2 technical repeats from 2 biological replicates, with variance of less than $8 \%$ for all samples. Values are scaled to direct neutral sugar analysis.

\begin{tabular}{|lcc|}
\hline Rhamnose Family & WT Col-0 & Triple urgt \\
2,3,4-Rha/Rha-t & 3.99 & \\
1,2-Rha & 88.72 & 80.16 \\
1,2,4-Rha & 0.60 & 0.30 \\
2,3,4-Rha & 0.16 & (Traces) \\
Total Rha & 93.47 & 83.92 \\
\hline Arabinose Family & & \\
2,3,5-Ara/ Ara-t & 1.34 & 2.12 \\
Total Ara & 1.34 & 2.12 \\
\hline
\end{tabular}




\begin{tabular}{|lll|} 
Xylose Family & & \\
2,3,4-Xyl/ Xyl-t & 0.69 & 2.35 \\
1,4-Xyl & 0.24 & 7.01 \\
1,2,4-Xyl & 0.30 & 0.58 \\
Total Xyl & 1.22 & 9.94 \\
\hline Galactose Family & & \\
2,3,4,6-Gal/ Gal-t & 0.36 & 0.46 \\
Total Gal & 0.36 & 0.46 \\
\hline Glucose Family & & \\
2,3,4,6-Glc/ Glc-t & 0.33 & 0.47 \\
2,3,6- Glc/ 4-Glc & 3.28 & 3.09 \\
Total Glc & 3.60 & 3.56 \\
\hline Manose Family & & \\
Total Man & n.d. & n.d. \\
\hline
\end{tabular}

\section{Figure Legends}

Figure 1: The six members of the URGT gene family are expressed during mucilage deposition

Top panel: schematic representation of seed coat epidermal cell differentiation in Arabidopis. Starch granules $(\mathrm{sg})$ are formed at 6 DAP and are surrounded by a large vacuole $(v)$ which pushes the cytoplasm (cy) to the cell periphery. At 8 DAP, mucilage polysaccharides are starting to be synthesized and deposited into the apoplast of epidermal cells. These force the cytoplasm into a central column filled with starch granules by 10 DAP due to the polar deposition of mucilage polysaccharides. At 12 DAP, mucilage deposition has stopped, and starch granules are degraded and replaced with wall material that forms a structure called the columella (c). 8 and 10 DAP are the stages when the production and accumulation of mucilage are maximal in the epidermal cells. M, mucilage; dw, distal wall; rw, radial wall.

Lower panel: RT-qPCR analysis of steady-state transcript levels in developing seeds from 6 to 12 days after pollination (DAP) for the six URGT genes in wild-type Col-0. The expression was calculated relative to EF1aA4 and a seed-specific reference gene 
(At4g12590). Error bars represent SE values from 3 biological replicates $(n=9)$. All the URGTs are expressed during epidermal cell differentiation.

Figure 2: Rhamnose content in the soluble mucilage of mutants with insertions in the 6 URGT genes

Soluble mucilage was extracted with water, hydrolyzed with TFA and Rha contents were determined by HPAEC. Bars represent the $\%$ of the Rha amount of the soluble mucilage relative to WT (100\%). Error bars represent the SE from 3 biological replicates $(5<n<6)$. Statistical analyses were performed using the Mann and Whitney test comparing to the wild-type with ${ }^{*}=p<0.05$ and ${ }^{* *}=p<0.01$. Schematic representation of seed mucilage layers is represented above the graph with $S M=$ soluble mucilage and $\mathrm{AM}=$ adherent mucilage. urgt2-1, urgt2-2, and urgt6-1 are knock out mutant lines while urgt4-2, urgt4-3, and urgt6-2 are knock-down mutant lines (see Supplemental Figure 4 and Parra-Rojas et al., 2019).

Figure 3: Changes in mucilage polysaccharide galacturonic acid content in urgt mutants

The galacturonic acid content of soluble and adherent mucilage from seeds of urgt mutant alleles. Polysaccharides from soluble mucilage (SM) were extracted with water, and adherent mucilage (AM) by enzyme digestion. GalA contents were quantified by the $p$-hydroxybyphenyl method. Error bars represent SE from 3 biological replicate $(n=3)$. Statistical analysis using the Mann and Whitney test comparing each line to the wild-type found no significant differences.

Figure 4: Galacturonic acid content of soluble and adherent mucilage layers of urgt single, double and triple mutant lines

Galacturonic acid content was determined using the m-hydroxybiphenyl method for polysaccharides in soluble mucilage (extracted with water) and adherent mucilage (by enzymatic digestion). Values represent means and SE from 4 biological replicates $(n=4)$ . Letters represents differences between different genotypes with statistical analyses performed using the Mann and Whitney test with $p<0.05$. 
Figure 5: urgt mutants have altered rhamnogalacturonan-I polymer length and numbers of molecules

(A) Intrinsic viscosity of outer soluble mucilage rhamnogalacturonan-I (RG-I) polymers in urgt mutants. Values represent means and SE from 4 biological replicates $(n=4)$.

(B) Number of RG-I molecules in urgt mutant lines. Values represent means and SE from 4 biological replicates $(n=4)$. A and $B$, letters represent differences between different genotypes with statistical analyses performed using the Mann and Whitney test with $p<0.05$.

Figure 6: Ratio between number of xyloses and number of RG-I molecules in the outer soluble mucilage layer of urgt mutants

The $N(X y l) / N(R G-I)$ ratio is similar in wild-type and urgt mutant lines. Values represent the means and error bars represent SE values from 4 biological replicates $(n=4)$. Letters represents differences between different genotypes with statistical analyses performed using the Mann and Whitney test with $p<0.05$. No differences were observed.

Figure 7: Immunolabelling of rhamnogalacturonan-I in adherent mucilage released from seeds of urgt2, urgt4 and urgt6 single, double and triple mutants INRA-RU1 labeled rhamnogalacturonan-I (RG-I) in the inner layer of adherent mucilage from mature seeds of wild-type and urgt mutants. Confocal microscopy optical section reconstructions of inner adherent mucilage (AM) released from imbibed seeds of wild-type (WT) Col-0, single, double and triple urgt mutants. Fluorescence corresponding to INRA-RU1 antibody labeling is green, and calcofluor white, used to detect $\beta-1,4-g l u c a n s$, is magenta. $A M$, adherent mucilage; $d w$, distal wall and $c$, columella. Bar $=50 \mu \mathrm{m}$.

Figure 8: Seeds of urgt mutants release adherent mucilage with differing porosity 
(A) Direct imbibition of dry seeds in FITC-dextran 70-kD molecules (orange). The extent that FITC-dextran molecules diffuse into the adherent mucilage (AM) depends on the latter's porosity. Images of seeds were digitally extracted and placed on the same background for adherent mucilage phenotype comparison. Scale bars $=50 \mu \mathrm{m}$. Quantification of $(B)$ the width of the AM layer and $(C)$ average intensity of yellow pixels over the inner adherent mucilage surface, using images of FITC-dextran staining. Values are means and SE values from 4 biological replicates. Measurements were carried out from more than 306 images for each genotype $(n>306$.) Letters represents differences between different genotypes with statistical analyses performed using the Tukey test with $\alpha=0.01$.

Figure 9: Relative gene expression of RRT1, GATL5, MUCI21, and UXT3 in the urgt triple mutant compared to the wild-type
$(\mathrm{A}-\mathrm{D})$,
$R G-I$
RHAMNOSYLTRANSFERASE1

(RRT1), GALACTURONOSYLTRANSFERASE-LIKE 5 (GATL5), MUCILAGE MODIFIED-21 (MUM5/MUCl21), and UDP-XYLOSE TRANSPORTER-3 UXT3 expression in developing seeds of the triple urgt mutant line urgt2-2 urgt4-2 urgt6-1 compared to its corresponding wild-type (Col-0 accession). Transcript abundance in developing seeds was determined by reverse transcription quantitative PCR (RT-qPCR) from 5 to 12 days after pollination (DAP), corresponding to stages when seed coat epidermal cell differentiation is in progress. Steady-state mRNA levels are represented as relative expression compared to two reference genes, EF1aA4 and the seed reference gene (At4g12590). Error bars represent SE values from 3 biological replicates $(n=9)$. Statistical analyses were performed using a Wilcoxon test and comparing to the wild-type, ${ }^{*} p<0.05 \%,{ }^{* *} p<0.01$.

Figure 10: Modulation of URGT2, URGT4, and URGT6 expression by MUM1, MYB61, TTG1, and GL2 transcription regulators

Comparison of URGT2, URGT4 and URGT6 expression in developing seeds of the wild-type (WT Col-0) to gl2-6, ttg2-3, mum1/luh-3, and myb61 mutants by reverse transcription quantitative PCR (RT-qPCR) analysis was used to determine the URGT2, 
URGT4 and URGT6 transcript levels in developing seeds at 8 days after pollination, which corresponds to the developmental stage when mucilage deposition is maximal in the wild-type. Steady-state mRNA levels are represented as relative expression compared to two reference genes, EF1aA4 and the seed reference gene (At4g12590). Error bars represent SE values from 3 biological replicates $(n=9)$. Statistical differences were analyzed using the Wilcoxon test and comparing to the wild-type, ${ }^{*} p<0.05 \%,{ }^{* *} p$ $<0.01$.

Figure 11: Hypothetical model of URGT2, URGT4, and URGT6 transcriptomic regulation by specific epidermal cell transcription factors

Expression levels observed in mutants indicate that TRANSPARENT TESTA GLABRA2 (TTG2), MYB61, GLABRA2 (GL2), and LEUNIG HOMOLOG/ MUCILAGE-MODIFIED1 $(L U H /$ MUM1) transcriptional regulators directly or indirectly control the expression of URGT2, URGT4, URGT6, UUAT1 and UUAT3. URGT2, URGT4, and URGT6 transport UDP-Rha, one of the principal substrates of RG-I, and are independently required for the elongation of soluble mucilage RG-I polymers. UUAT1 and UUAT3 transport UDP-Uronic acid and undetermined UDP-Sugars that are also required for the synthesis of mucilage pectin (Saez-Aguayo et al., 2017; Parra-Rojas et al., 2019). URGT2, URGT6, URGT4, UUAT1, UUAT3, also act in adherent mucilage structuration. White proteins: Transcription factors described as acting on mucilage secretory cells (MSC) development. Doted proteins: UDP-Sugar Transporters regulated by Transcription Factors (gray proteins) evaluated in this study. Colored and dotted arrows indicate regulation demonstrated here. MSC, mucilage secretory cells.

\section{References}

Alonso JM, Stepanova AN, Leisse TJ, Kim CJ, Chen H, Shinn P, Stevenson DK, Zimmerman J, Barajas P, Cheuk R, et al. 2003. Genome-wide insertional mutagenesis of Arabidopsis thaliana. Science. 301, 653-657.

Arsovski AA, Haughn GW, Western TL. 2010. Seed coat mucilage cells of 
Arabidopsis thaliana as a model for plant cell wall research. Plant Signaling \& Behaviour. 5, 796-801.

Atmodjo MA, Hao Z, Mohnen D. 2013. Evolving Views of Pectin Biosynthesis. Annual Review of Plant Biology. 64, 747-779.

Bassel GW, Fung P, Freeman Chow T-f, Foong JA, Provart NJ, Cutler SR. 2008. Elucidating the Germination Transcriptional Program Using Small Molecules. Plant Physiology. 147, 143-155.

Blakeney AB, Harris PJ, Henry RJ, Stone BA. 1983. A simple and rapid preparation of alditol acetates for monosaccharide analysis, Carbohydrate Researc. 113, 291-299.

Brent LR, Malcolm AO, Debra M. 2001. Pectins: structure, biosynthesis, and oligogalacturonide-related signaling, Phytochemistry. 57, 929-967.

Carpita, NC. and Gibeaut, DM. 1993. Structural models of primary cell walls in flowering plants: consistency of molecular structure with the physical properties of the walls during growth. The Plant Journal. 3, 1-30.

Clough SJ and Bent AF. 1998. Floral dip: a simplified method for Agrobacterium mediated transformation of Arabidopsis thaliana. The Plant Journal.16: 735-743.

Cosgrove DJ. 2016. Plant cell wall extensibility: connecting plant cell growth with cell wall structure, mechanics, and the action of wall-modifying enzymes, Journal of Experimental Botany. 67, 463-476.

Dean GH, Zheng H, Tewari J, Huang J, Young DS, Hwang YT, Western TL, Carpita NC, McCann MC, Mansfield SD, Haughn GW. 2007. The Arabidopsis MUM2 gene encodes a $\beta$-galactosidase required for the production of seed coat mucilage with correct hydration properties. Plant Cell. 19, 4007-4021.

Ebert B, Rautengarten C, Guo X, Xiong G, Stonebloom S, Smith-Moritz AM, Herter T, Chan LJ, Adams PD, Petzold CJ, et al. 2015. Identification and characterization of 
a Golgi-localized UDP-xylose transporter family from Arabidopsis. Plant Cell. 27, 1218-1227.

Fabrissin I, Cueff G, Berger A, Granier F, Sallé C, Poulain D, Ralet M-C, North H.M et al. 2019. Natural variation reveals a key role for rhamnogalacturonan I in seed outer mucilage and underlying genes. Plant Physiol. pp.00763.2019. doi: 10.1104/pp.19.00763.

Francoz E, Ranocha P, Burlat V, Dunand C. 2015. Arabidopsis seed mucilage secretory cells: regulation and dynamics. Trends Plant Science. 20, 515-524.

Golz JF, Allen PJ, Li SF, Parish RW, Jayawardana NU, Bacic A, et al. 2018. Layers of regulation - Insights into the role of transcription factors controlling mucilage production in the Arabidopsis seed coat. Plant Sci. 272, 179-192.

Hong SM, Bahn SC, Lyu A, Jung HS, Ahn JH. 2010. Identification and testing of superior reference genes for a starting pool of transcript normalization in Arabidopsis. Plant Cell Physiology 51, 1694-1706.

Huang J, DeBowles D, Esfandiari E, Dean G, Carpita NC, Haughn GW. 2011. The Arabidopsis transcription factor LUH/MUM1 is required for extrusion of seed coat mucilage. Plant Physiology. 156, 491-502.

Hu R, Li J, Wang X, Zhao X, Yang X, Tang Q, He G, Zhou G, Kong Y. 2016. Xylan synthesized by Irregular Xylem 14 (IRX14) maintains the structure of seed coat mucilage in Arabidopsis. Journal of Experimental Botany. 67, 1243-1257.

Ishida T, Hattori S, Sano R, Inoue K, Shirano Y, Hayashi H, Shibata D, Sato S, Kato T, Tabata S, et al. 2007. Arabidopsis TRANSPARENT TESTA GLABRA2 is directly regulated by R2R3 MYB transcription factors and is involved in regulation of GLABRA2 transcription in epidermal differentiation. Plant Cell. 19, 2531-2543.

Knappe S, Flügge UI, Fischer K. 2003. Analysis of the plastidic phosphate translocator gene family in Arabidopsis and identification of new phosphate 
translocator-homologous transporters, classified by their putative substrate-binding site. Plant Physiology.131, 1178-1190.

Kong Y, Zhou G, Abdeen AA, et al. 2013. GALACTURONOSYLT RANSFERASE-LIKE5 is involved in the production of Arabidopsis seed coat mucilage. Plant Physiology. 163: 1203-1217.

Le BH, Cheng C, Bui AQ, Wagmaister JA, Henry KF, Pelletier J, Kwong L, Belmonte M, Kirkbride R, Horvath S, Drews GN, Fischer RL, Okamuro JK, Harada JJ, ... Goldberg RB. 2010. Global analysis of gene activity during Arabidopsis seed development and identification of seed-specific transcription factors. Proceedings of the National Academy of Sciences of the United States of America. 107(18), 8063-70.

Levesque-Tremblay G, Pelloux J, Braybrook SA, Müller K. 2015. Tuning of pectin methylesterification: consequences for cell wall biomechanics and development. Planta. 242, 791-811.

Macquet A, Ralet MC, Kronenberger J, Marion-Poll A, North HM. 2007a. In situ, chemical and macromolecular study of the composition of Arabidopsis thaliana seed coat mucilage. Plant Cell Physiology. 48: 984-999.

Macquet A, Ralet MC, Loudet O, Kronenberger J, Mouille G, Marion-Poll, A, and North HM. 2007b. A naturally occurring mutation in an Arabidopsis accession affects a beta-D-galactosidase that increases the hydrophilic potential of rhamnogalacturonan I in seed mucilage. Plant Cell. 19, 3990-4006.

Mohnen D. 2008. Pectin structure and biosynthesis, Current Opinion in Plant Biology. $11,266-277$.

Nakagawa T, Nakamura S, Tanaka K, Kawamukai M, Suzuki T, Nakamura K, Kimura T, Ishiguro S. 2008. Development of R4 Gateway Binary Vectors (R4pGWB) Enabling High-Throughput Promoter Swapping for Plant Research. Bioscience, Biotechnology, and Biochemistry. 72:2, 624-629.

North HM, De Almeida A, Boutin J-P, Frey A, To A, Botran L, Sotta B, Marion-Poll 
A. 2007. The Arabidopsis ABA- deficient mutant aba4 demonstrates that the major route for stress-induced $A B A$ accumulation is via neoxanthin isomers. Plant Journal 50 , 810-824.

North HM, Berger A, Saez-Aguayo S, Ralet M-C. 2014. Understanding polysaccharide production and properties using seed coat mutants: future perspectives for the exploitation of natural variants. Annals of Botany. 114: 1251-1263.

Orellana A, Moraga C, Araya M, Moreno A. 2016. Overview of nucleotide sugar transporter gene family functions across multiple species. Journal of Molecular Biology. 428, 3150-3165.

Parra Rojas JP, Largo-Gosens A, Carrasco T, Celiz-Balboa J, Temple H, Sanhueza D, Reyes FC, Meneses C, Saez-Aguayo S, and Orellana A. 2019. New steps in mucilage biosynthesis revealed by analysis of the transcriptome of UDP-rhamnose/UDP-galactose transporter 2 (URGT2) mutant. Journal of Experimental Botany. 70, 5071-5088.

Ralet M-C, Tranquet O, Poulain D, Moïse A, Guillon F. 2010. Monoclonal antibodies to rhamnogalacturonan I backbone. Planta. 231, 1373-1383.

Ralet M-C, Crépeau MJ, Vigouroux J, Tran J, Berger A, Sallé C, Granier F, Botran L, North HM. 2016. Xylans provide the structural driving force for mucilage adhesion to the Arabidopsis seed coat. Plant Physiology. 171, 165-178.

Rasool B, McGowan J, Pastok D, Marcus SE, Morris J, Verrall SR, Hedley PE, Hancock RD, Foyer, CH. 2017. Redox control of aphid resistance through altered cell wall composition and nutritional quality. Plant Physiology.175, 259-271.

Rautengarten C, Ebert B, Moreno I, Temple H, Herter T, Link B, Doñas-Cofré D, Moreno A, Saez-Aguayo S, Blanco F, et al. 2014. The Golgi localized bifunctional UDP- rhamnose/UDP-galactose transporter family of Arabidopsis. Proceedings of the National Academy of Sciences USA .111, 11563-11568. 
Reyes F, Orellana A. 2008. Golgi transporters: opening the gate to cell wall polysaccharide biosynthesis. Current Opinion of Plant Biology. 11, 244-251.

Saez-Aguayo S, Ralet M-C, Berger A, Botran L, Ropartz D, Marion-Poll A, North HM. 2013. PECTIN METHYLESTERASE INHIBITOR6 promotes Arabidopsis mucilage release by limiting methylesterification of homogalacturonan in seed coat epidermal cells. Plant Cell. 25, 308-323.

Saez-Aguayo S, Rondeau-Mouro C, Macquet A, Kronholm I, Ralet MC, Berger A, Sallé C, Poulain D, Granier F, Botran L, Loudet O, de Meaux J, Marion-Poll A, North HM. 2014. Local evolution of seed flotation in Arabidopsis. PLoS Genet. 2014;10(3):e1004221. doi:10.1371/journal.pgen.1004221

Saez-Aguayo S, Rautengarten C, Temple H, Sanhueza D, Ejsmentewicz T, Sandoval-lbañez O, Doñas D, Parra-Rojas JP, Ebert B, Lehner A, et al. 2017. UUAT1 is a Golgi-localized UDP-uronic acid transporter that modulates the polysaccharide composition of Arabidopsis seed mucilage. Plant Cell. 29. 129-143.

Schindelin J, Ruede CT, Hiner MC, Eliceiri KW. 2015. The ImageJ ecosystem: An open platform for biomedical image analysis. Molecular reproduction and development. 82, 518-29.

Shi D, Ren A, Tang X, Qi G, Xu Z, Chai G, Hu R, Zhou G, Kong Y. 2018. MYB52 negatively regulates pectin demethylesterification in seed coat mucilage. Plant Physiology. 176, 2737-2749.

Sullivan, S., Ralet, M.-C., Berger, A., Diatloff, E., Bischoff, V., Gonneau, M., Marion-Poll, A., and North, H.M. 2011. CESA5 is required for the synthesis of cellulose with a role in structuring the adherent mucilage of Arabidopsis seeds. Plant Physiol. 156: 1725-1739.

Tan L, Eberhard S, Pattathil S, Warder C, Glushka J, Yuan C, Hao Z, Zhu X, Avci U, Miller J.S, Baldwin D, Pham C, Orlando R, Darvill A, Hahn M.G, Kieliszewski 
M.J , Mohnen D. 2013. An Arabidopsis Cell Wall Proteoglycan Consists of Pectin and Arabinoxylan Covalently Linked to an Arabinogalactan Protein. The Plant Cell Jan 2013, 25 (1) $270-287$.

Takenaka Y, Kato K, Ogawa-Ohnishi M, Tsuruhama K, Kajiura H, Yagyu K, Takeda A, Takeda Y, Kunueda T, Hara-Nishimura I, Kuroha T, Nishitani K, Matsubqyashi Y, Ishimizu T. 2018. Pectin RG-I rhamnosyltransferases represent a novel plant-specific glycosyltransferase family. Nat Plants. 4, 669-676.

Takenaka Y, Watanabe Y, Schuetz M, Unda F, Hill JL, Phookaew P, Yoneda A, Mansfield SD, Samuels L, Ohtani M, Demura T. 2018. Patterned Deposition of Xylan and Lignin is Independent from that of the Secondary Wall Cellulose of Arabidopsis Xylem Vessels. The Plant Cell, 30, 2663-2676.

Temple H, Saez-Aguayo S, Reyes FC, Orellana A. 2016. The inside and outside: topological issues in plant cell wall biosynthesis and the roles of nucleotide sugar transporters. Glycobiology. 26, 913-925.

Thibault, JE. 1979 .Automisation du dosage des subtances pectiques par la methode au meta-hidroxydiphenyl. Lebensm.-Wiss. Technol. 12, 247.

Verhertbruggen Y, Marcus SE, Chen J, Knox JP. 2013. Cell wall pectic arabinans influence the mechanical properties of Arabidopsis thaliana inflorescence stems and their response to mechanical stress. Plant Cell Physiology. 54, 1278-1288.

Voiniciuc C, Yang B, Schmidt MH, Günl M, Usadel B. 2015a. Starting to gel: how Arabidopsis seed coat epidermal cells produce specialized secondary cell walls. Integrative Journal of Molecular Sciences. 16, 3452-3473.

Voiniciuc C, Schmidt MH, Berger A, Yang B, Ebert B, Scheller HV, North HM, Usadel B, Günl M. 2015b. MUCILAGE-RELATED10 produces galactoglucomannan that maintains pectin and cellulose architecture in Arabidopsis seed mucilage. Plant Physiology. 169, 403-420. 
Voiniciuc C, Günl M, Schmidt MH, Usadel B. 2015c. Highly branched xylan made by IRREGULAR XYLEM14 and MUCILAGE-RELATED21 links mucilage to Arabidopsis seeds. Plant Physiology. 169, 2481-2495.

Voiniciuc C, Engle KA, Günl M, Dieluweit S, Heinrich-Wilhelm MS,Yang JY, Moremen WK, Mohnen D, Usadel B. 2018. Identification of Key Enzymes for Pectin Synthesis in Seed Mucilage. Plant Physiology. 178, 1045-1064.

Walker M, Tehseen M, Doblin MS, Pettolino FA, Wilson SM, Bacic A, Golz JF. 2011. The transcriptional regulator LEUNIG_HOMOLOG regulates mucilage release from the Arabidopsis testa. Plant Physiology. 156: 46-60.

Western TL, Skinner DJ, Haughn GW. 2000. Differentiation of mucilage secretory cells of the Arabidopsis seed coat. Plant Physiology. 122, 345-356.

Western TL, Young DS, Dean GH, Tan WL, Samuels AL, Haughn GW. 2004. MUCILAGE-MODIFIED4 encodes a putative pectin biosynthetic enzyme developmentally regulated by APETALA2, TRANSPARENT TESTA GLABRA1, and GLABRA2 in the Arabidopsis seed coat. Plant Physiology 134, 296-306.

Western TL. 2012. The sticky tale of seed coat mucilages: production, genetics, and role in seed germination and dispersal. Seed Science Research. 22, 1-25.

Williams M, Cornuault V, Irani AH, Vaughan Symonds V, Malmstrom J, An Y, Sims IA, Carnachan SM, Sallé C, and North HM. 2020. Polysaccharide Structures in the Outer Mucilage of Arabidopsis Seeds Visualised by AFM. Biomacromolecules. DOI: 10.1021/acs.biomac.9b0175.

Willats WG, McCarnety L, Mackie W, Knox JP. 2001. Pectin: cell biology and prospects for functional analysis. Plant Mol. Bio. 47, 9-27.

Winter D, Vinegar B, Nahal H, Ammar R, Wilson GV, Provart, NJ. 2007. An "Electronic Fluorescent Pictograph" browser for exploring and analyzing large-scale biological data sets. PloS one. 2(8), e718. 
Wolf S. 2017. Plant cell wall signalling and receptor-like kinases. Biochemical Journal. 474, 471-492.

Young RE, McFarlane HE, Hahn MG, Western TL, Haughn GW, Samuels AL. 2008. Analysis of the Golgi apparatus in Arabidopsis seed coat cells during polarized secretion of pectin-rich mucilage. Plant Cell. 20, 1623-1638.

Zhao X, Qiao L, Wu AM. 2017. Effective extraction of Arabidopsis adherent seed mucilage by ultrasonic treatment. Scientific Reports. 7, 40672. 


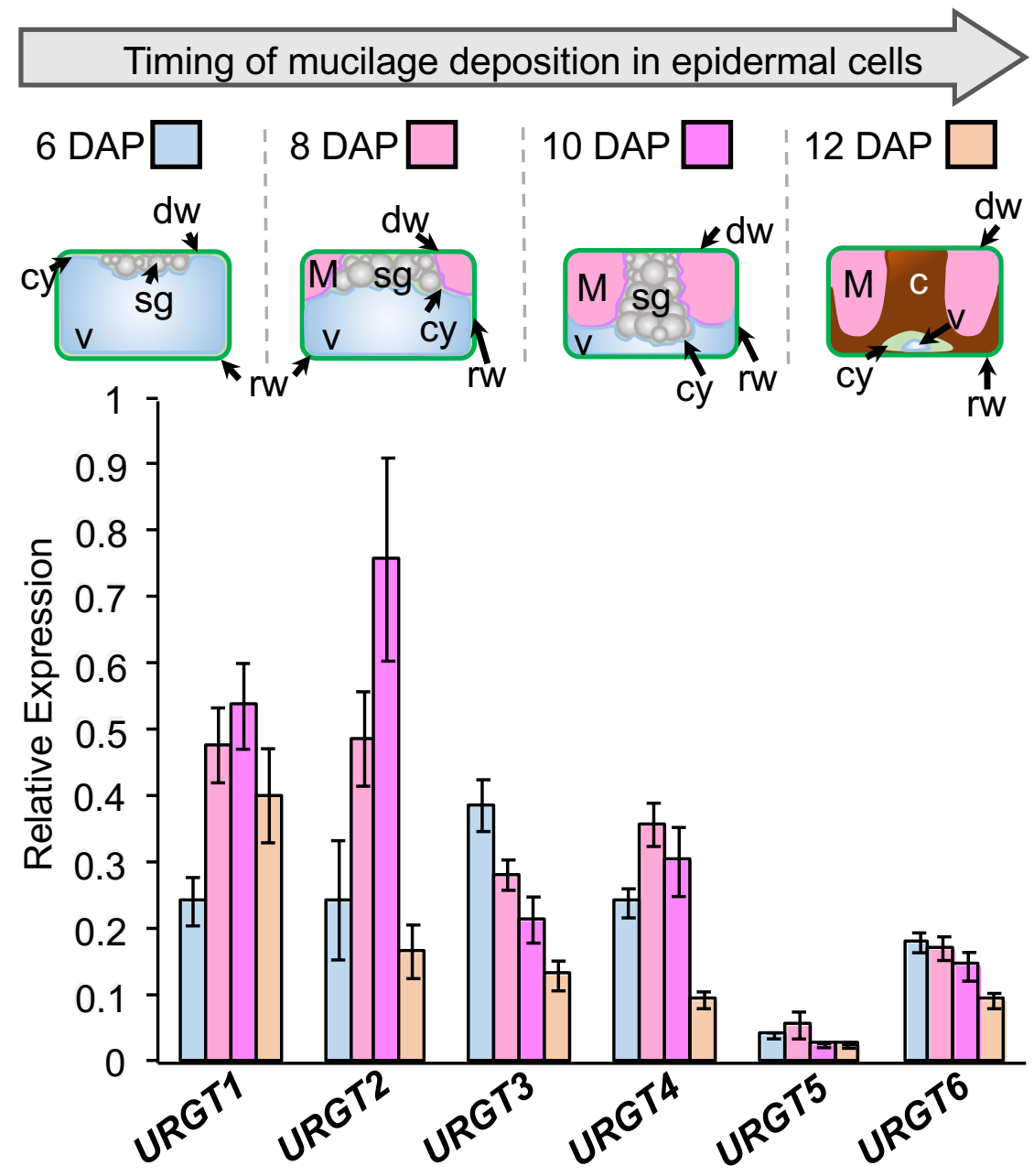

Figure 1: The six members of the URGT gene family are expressed during mucilage deposition

Top panel: schematic representation of seed coat epidermal cell differentiation in Arabidopis. Starch granules (sg) are formed at 6 DAP and are surrounded by a large vacuole (v) which pushes the cytoplasm (cy) to the cell periphery. At 8 DAP, mucilage polysaccharides are starting to be synthesized and deposited into the apoplast of epidermal cells. These force the cytoplasm into a central column filled with starch granules by 10 DAP due to the polar deposition of mucilage polysaccharides. At 12 DAP, mucilage deposition has stopped, and starch granules are degraded and replaced with wall material that forms a structure called the columella (c). 8 and 10 DAP are the stages when the production and accumulation of mucilage are maximal in the epidermal cells. M, mucilage; dw, distal wall; rw, radial wall.

Lower panel: RT-qPCR analysis of steady-state transcript levels in developing seeds from 6 to 12 days after pollination (DAP) for the six URGT genes in wild-type Col-0. The expression was calculated relative to EF1aA4 and a seed-specific reference gene (At4g12590). Error bars represent SE values from 3 biological replicates $(n=9)$. All the URGTs are expressed during epidermal cell differentiation. 


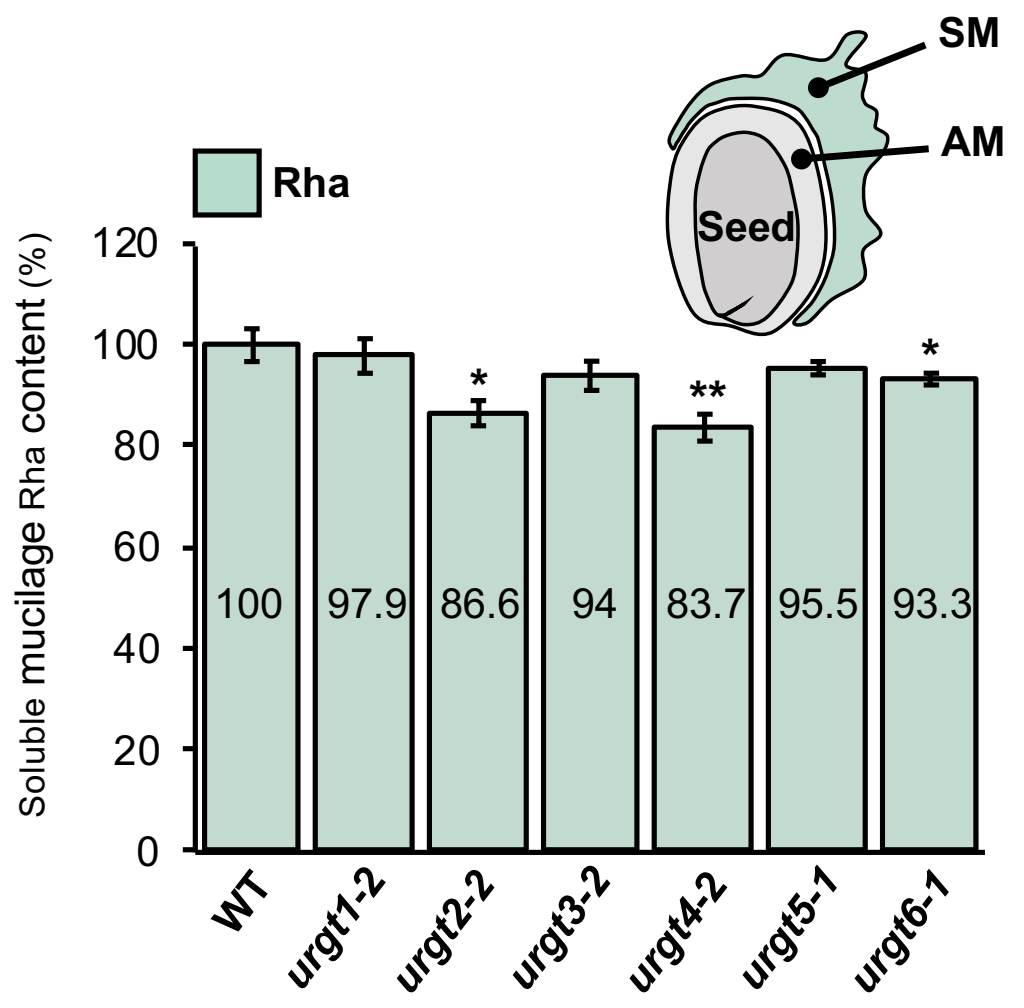

Figure 2: Rhamnose content in the soluble mucilage of mutants with insertions in the 6 URGT genes

Soluble mucilage was extracted with water, hydrolyzed with TFA and Rha contents were determined by HPAEC. Bars represent the \% of the Rha amount of the soluble mucilage relative to WT $(100 \%)$. Error bars represent the SE from 3 biological replicates $(5<n<6)$. Statistical analyses were performed using the Mann and Whitney test comparing to the wild-type with ${ }^{*}=p<0.05$ and ${ }^{* *}=p<0.01$. Schematic representation of seed mucilage layers is represented above the graph with $\mathrm{SM}=$ soluble mucilage and $\mathrm{AM}=$ adherent mucilage. urgt2-1, urgt2-2, and urgt6-1 are knock out mutant lines while urgt4-2, urgt4-3, and urgt6-2 are knock-down mutant lines (see Supplemental Figure 4 and Parra-Rojas et al., 2019). 


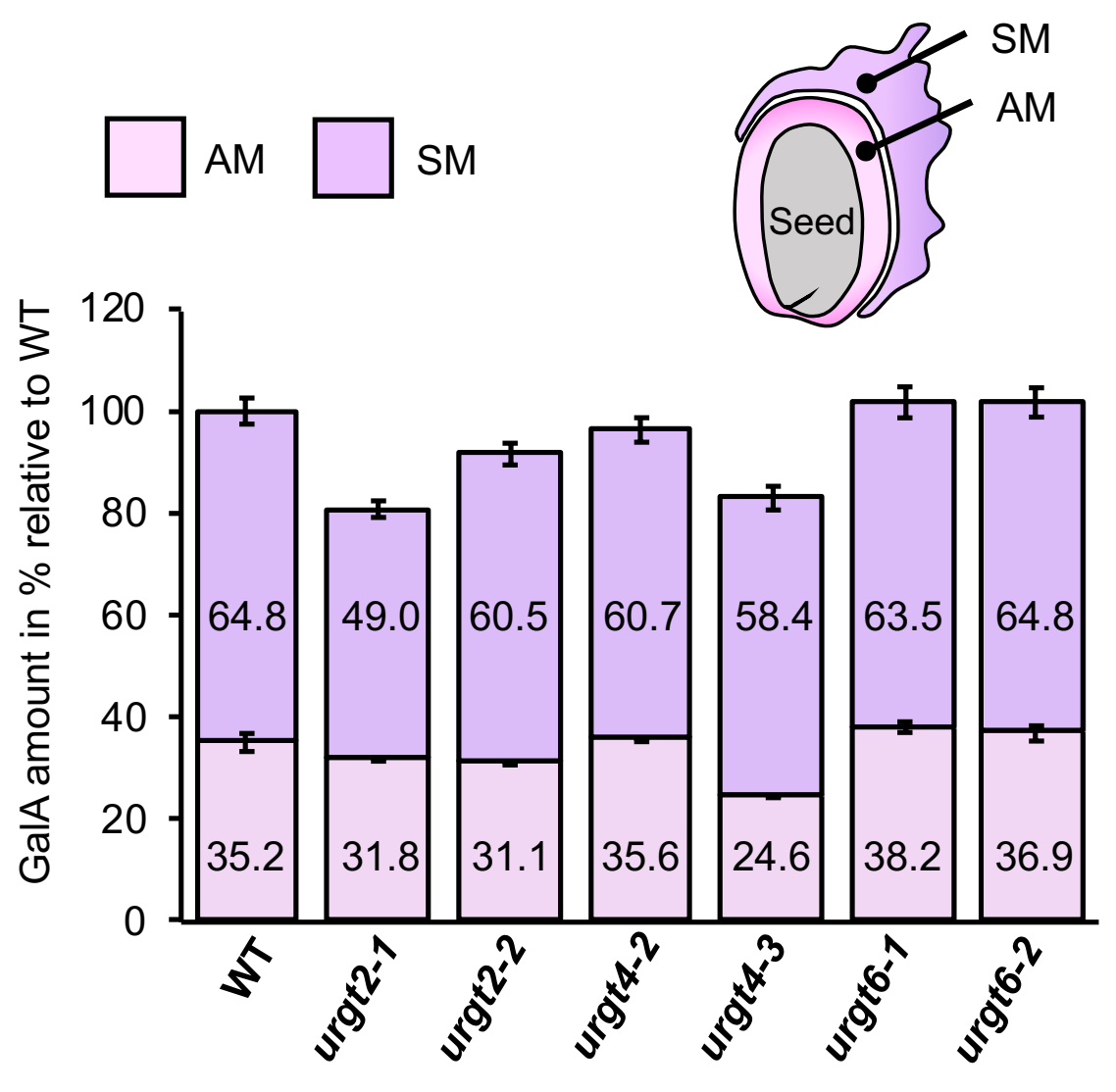

Figure 3: Changes in mucilage polysaccharide galacturonic acid content in urgt mutants

The galacturonic acid content of soluble and adherent mucilage from seeds of urgt mutant alleles. Polysaccharides from soluble mucilage (SM) were extracted with water, and adherent mucilage (AM) by enzyme digestion. GalA contents were quantified by the phydroxybyphenyl method. Error bars represent SE from 3 biological replicate $(n=3)$. Statistical analysis using the Mann and Whitney test comparing each line to the wildtype found no significant differences. 


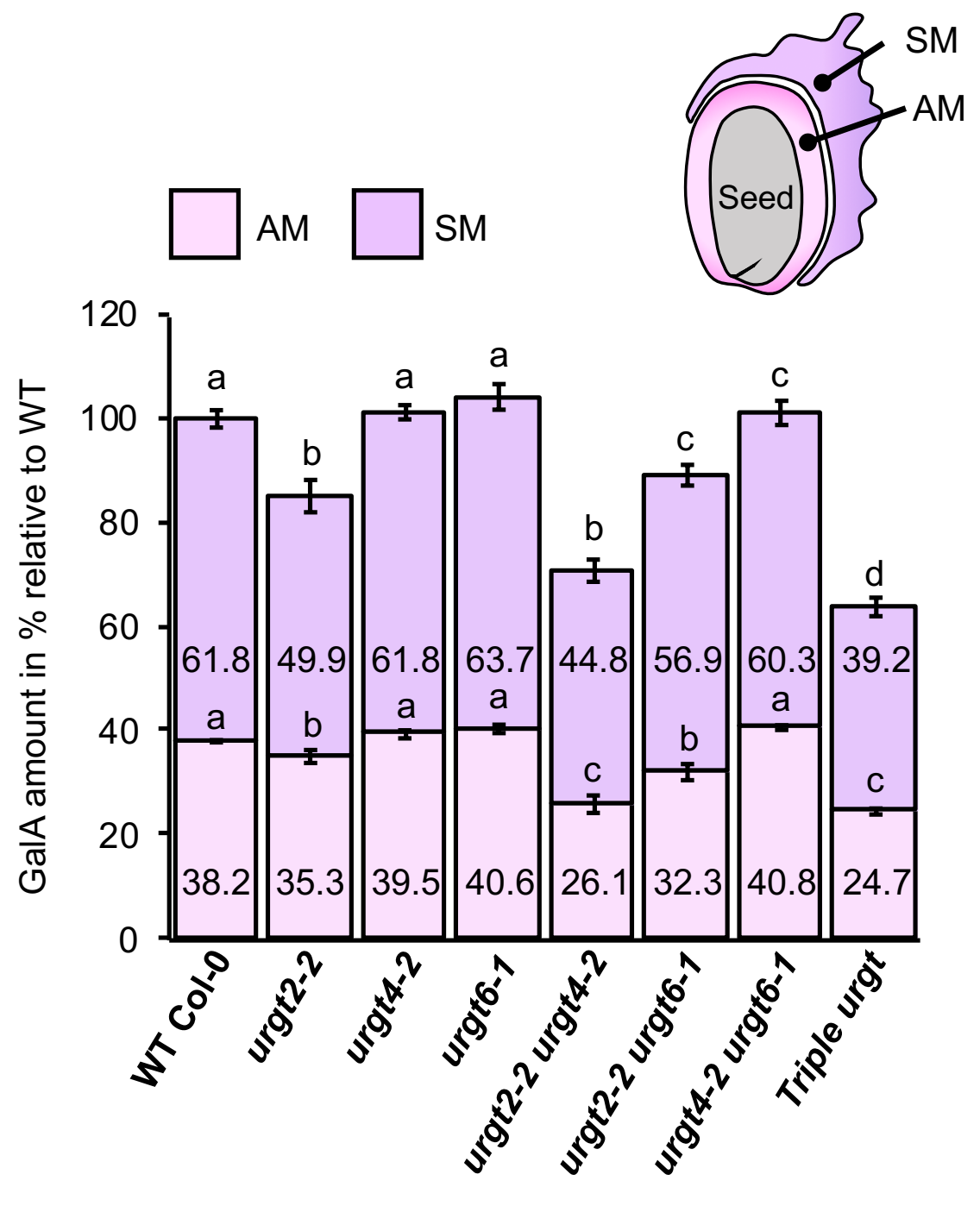

Figure 4: Galacturonic acid content of soluble and adherent mucilage layers of urgt single, double and triple mutant lines

Galacturonic acid content was determined using the mhydroxybiphenyl method for polysaccharides in soluble mucilage (extracted with water) and adherent mucilage (by enzymatic digestion). Values represent means and SE from 4 biological replicates $(n=4)$. Letters represents differences between different genotypes with statistical analyses performed using the Mann and Whitney test with $p<0.05$. 
A

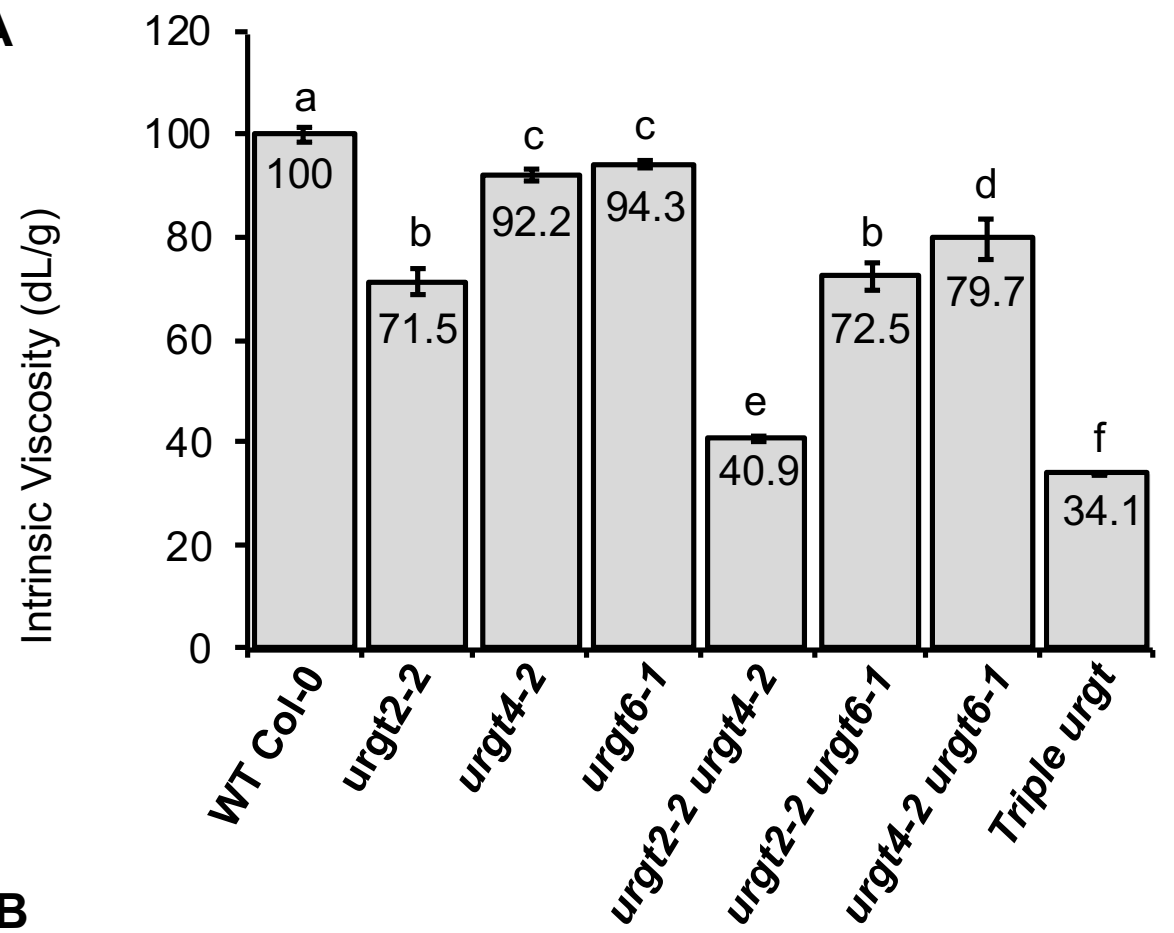

B

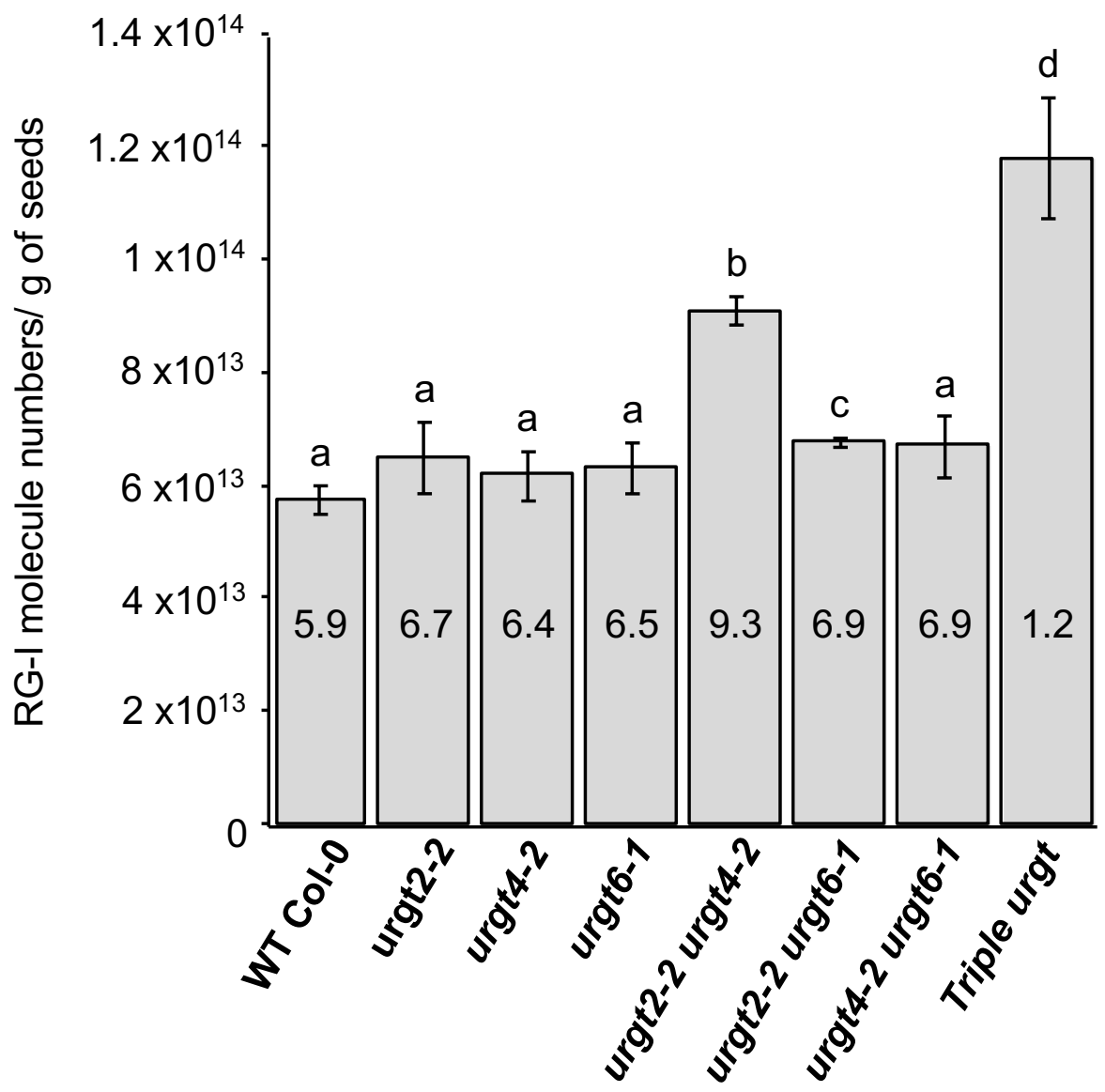

Figure 5: urgt mutants have altered rhamnogalacturonan-I polymer length and numbers of molecules

(A) Intrinsic viscosity of outer soluble mucilage rhamnogalacturonan-I (RG-I) polymers in urgt mutants. Values represent means and SE from 4 biological replicates $(n=4)$.

(B) Number of RG-I molecules in urgt mutant lines. Values represent means and SE from 4 biological replicates $(n=4)$. A and $B$, letters represent differences between different genotypes with statistical analyses performed using the Mann and Whitney test with $p<0.05$. 


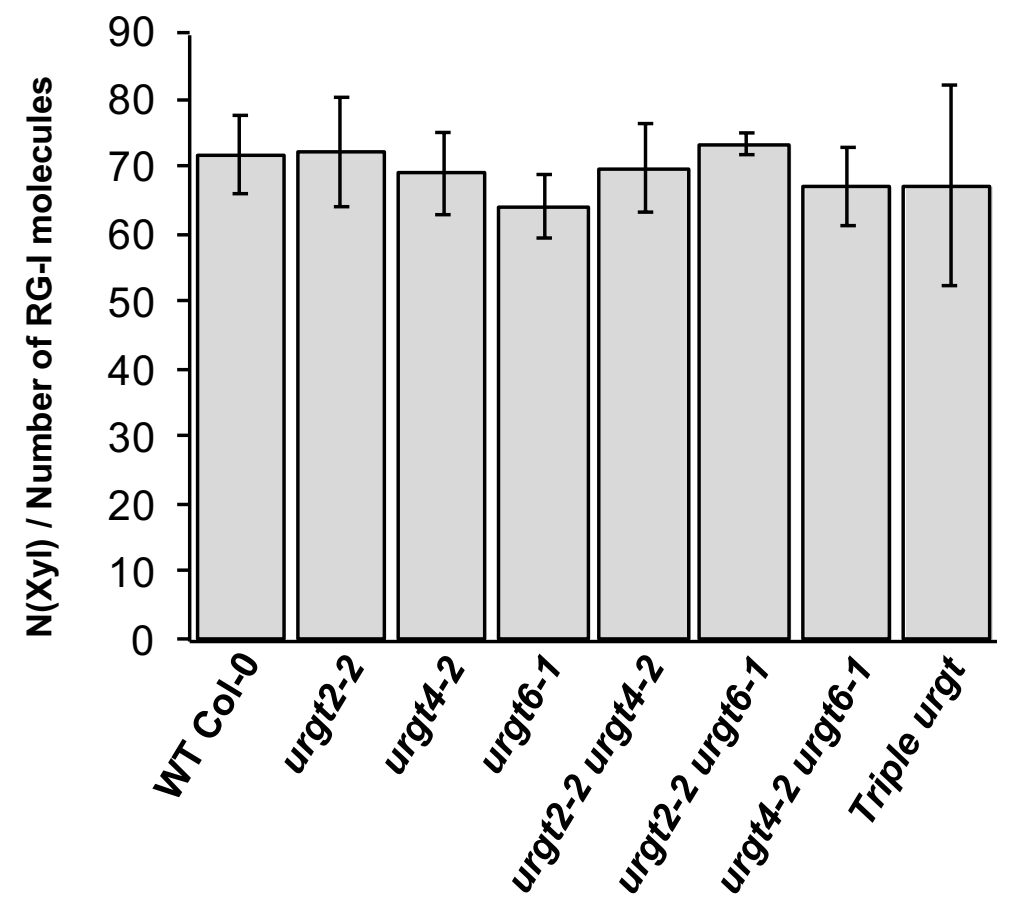

Figure 6: Ratio between number of xyloses and number of RG-I molecules in the outer soluble mucilage layer of urgt mutants

The $\mathrm{N}(\mathrm{Xyl}) / \mathrm{N}(\mathrm{RG}-\mathrm{I})$ ratio is similar in wild-type and urgt mutant lines. Values represent the means and error bars represent SE values from 4 biological replicates $(n=4)$. Letters represents differences between different genotypes with statistical analyses performed using the Mann and Whitney test with $p<0.05$. No differences were observed. 


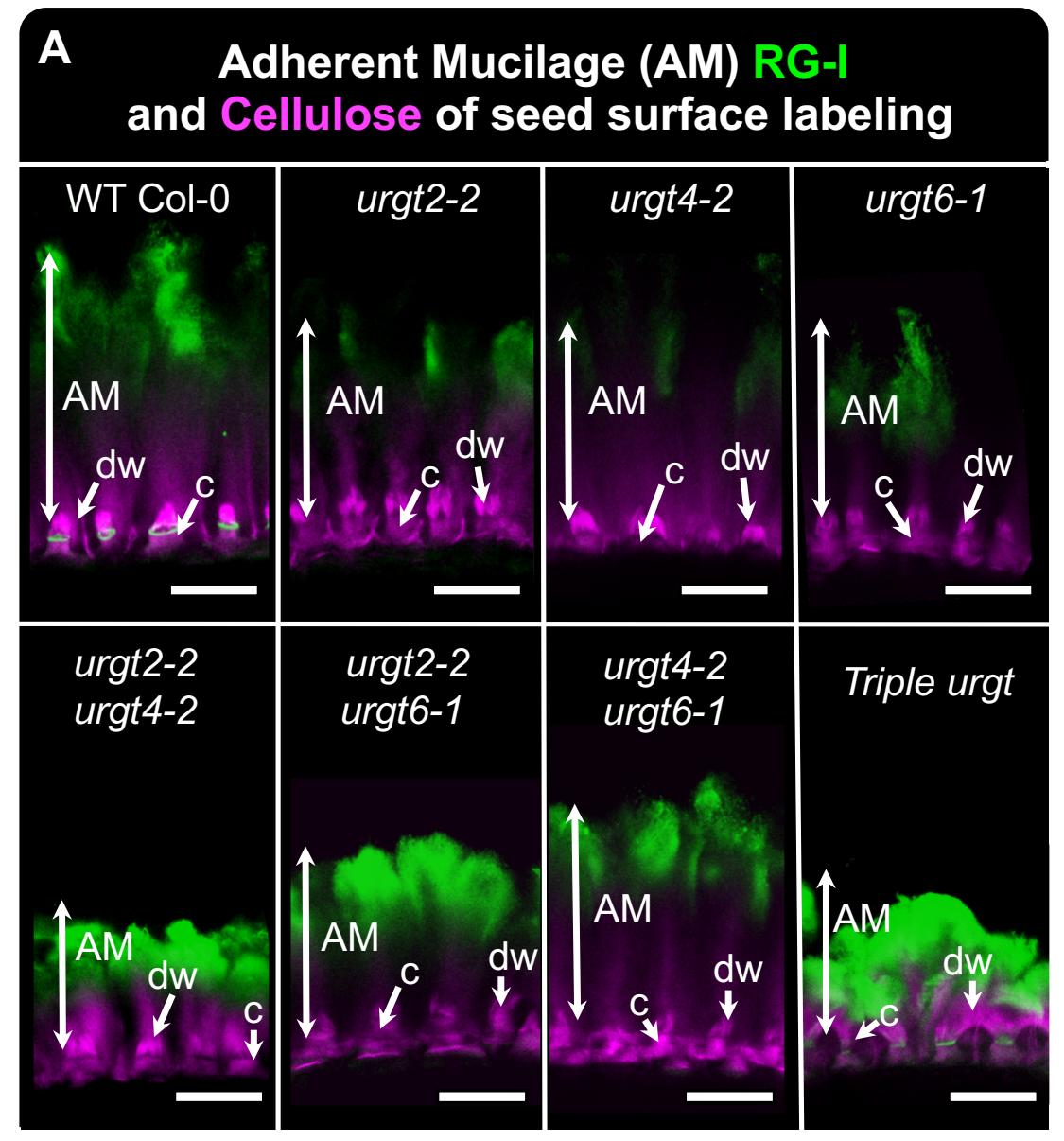

Figure 7: Immunolabelling of rhamnogalacturonan-I in adherent mucilage released from seeds of urgt2, urgt4 and urgt 6 single, double and triple mutants

INRA-RU1 labelled rhamnogalacturonan I (RG-I) in the inner layer of adherent mucilage from mature seeds of wild-type and urgt mutants. Confocal microscopy optical section reconstructions of inner adherent mucilage (AM) released from imbibed seeds of wild-type (WT) Col-0, single, double and triple urgt mutants. Fluorescence corresponding to INRA RU1 antibody labeling is green, and calcofluor white, used to detect $\beta-1,4$-glucans, is magenta. $\mathrm{AM}$, adherent mucilage; $d w$, distal wall and $\mathrm{c}$, columella. Bar $=50 \mu \mathrm{m}$ 

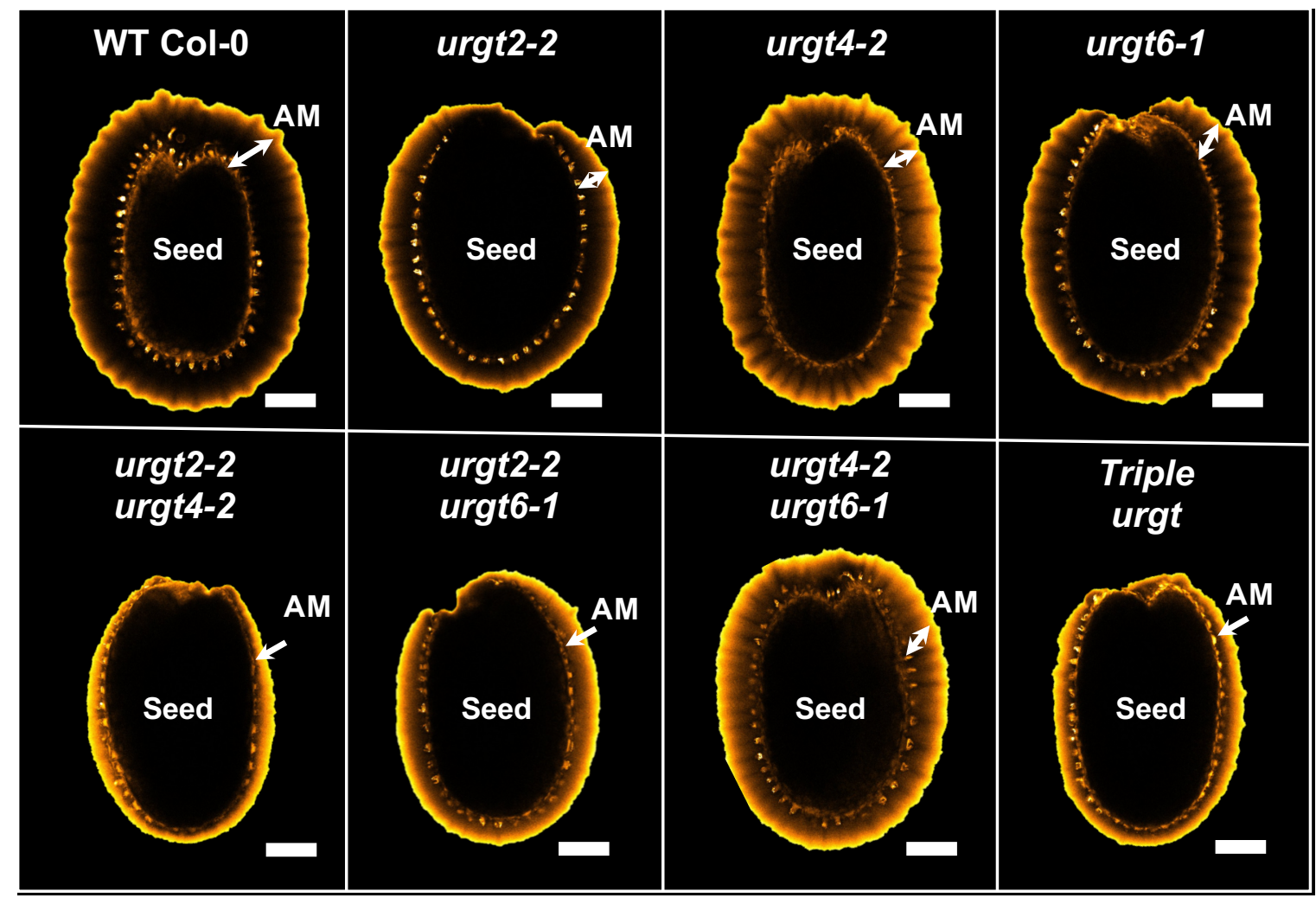

B

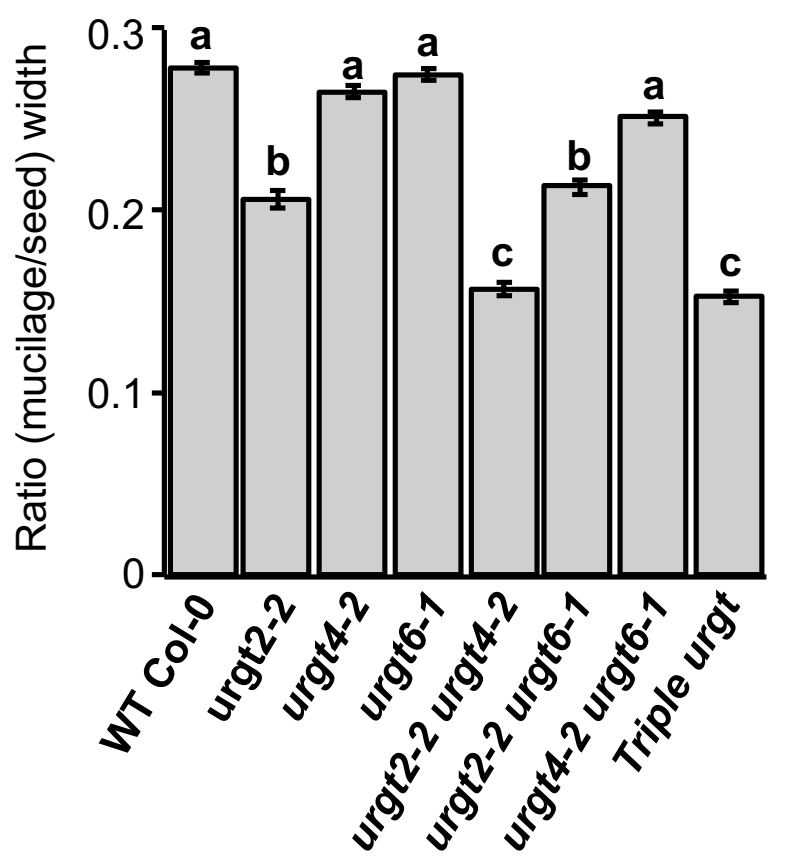

C

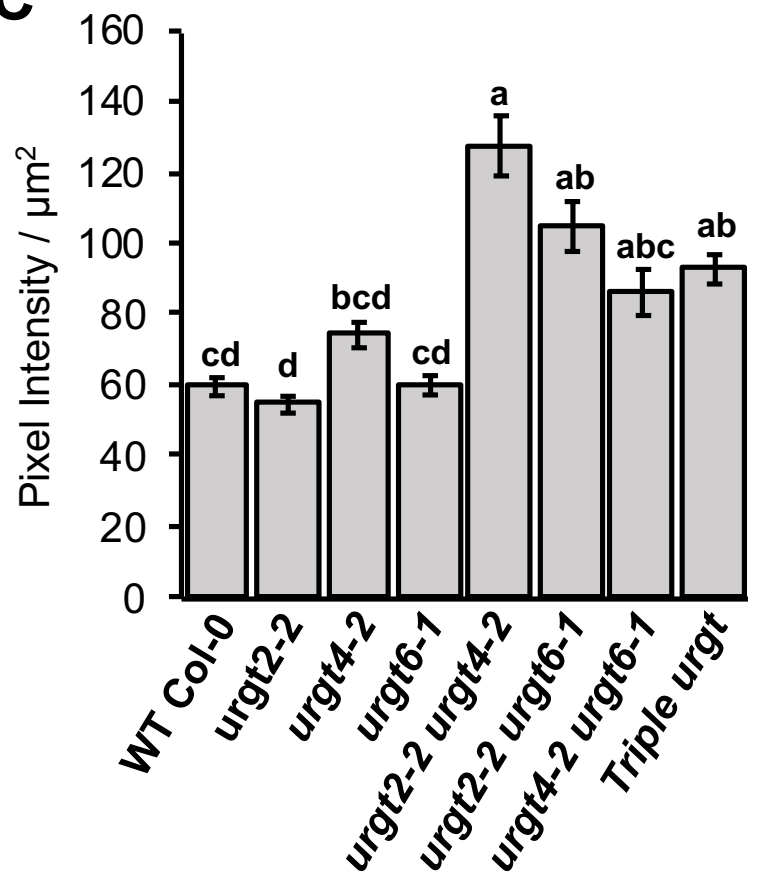

Figure 8: Seeds of urgt mutants release adherent mucilage with differing porosity

(A) Direct imbibition of dry seeds in FITC-dextran 70-kD molecules (orange). The extent that FITC-dextran molecules diffuse into the adherent mucilage (AM) depends on the latter's porosity. Images of seeds were digitally extracted and placed on the same background for adherent mucilage phenotype comparison. Scale bars $=50 \mu \mathrm{m}$. Quantification of (B) the width of the AM layer and (C) average intensity of yellow pixels over the inner adherent mucilage surface, using images of FITC-dextran staining. Values are means and SE values from 4 biological replicates. Measurements were carried out from more than 306 images for each genotype $(n>306$.) Letters represents differences between different genotypes with statistical analyses performed using the Tukey test with $\alpha=0.01$. 


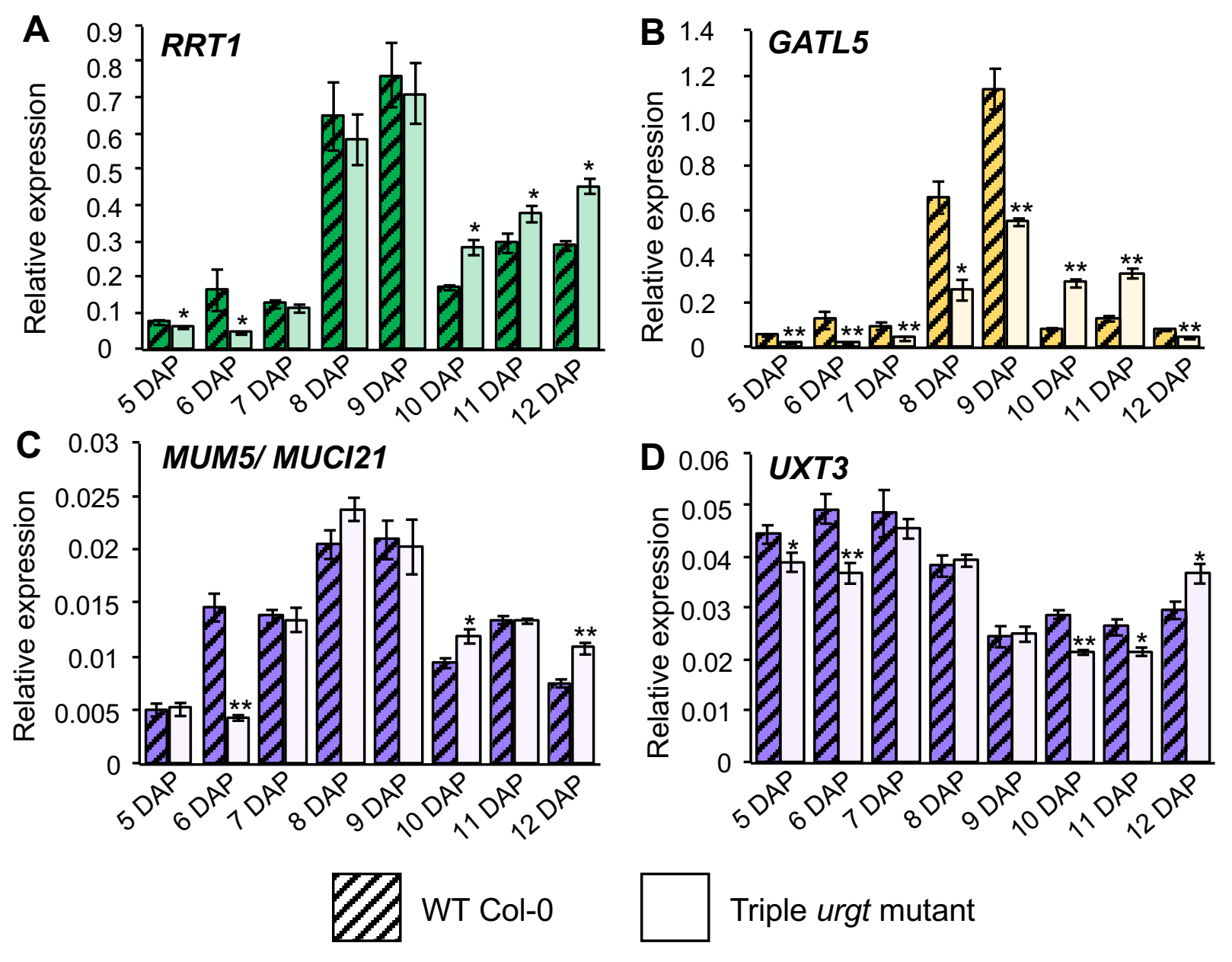

Figure 9: Relative gene expression of RRT1, GATL5, $M U C / 21$ and $U X T 3$ in the urgt triple mutant compared to the wild-type

(A-D), RG-I RHAMNOSYLTRANSFERASE1 (RRT1), GALACTURONOSYLTRANSFERASE-LIKE 5 (GATL5), MUCILAGE MODIFIED-21 (MUM5/MUCI21), and UDPXYLOSE TRANSPORTER-3 UXT3 expression in developing seeds of the triple urgt mutant line urgt2-2 urgt4-2 urgt6-1 compared to its corresponding wild-type (Col-0 accession). Transcript abundance in developing seeds was determined by reverse transcription quantitative PCR (RT-qPCR) from 5 to 12 days after pollination (DAP), corresponding to stages when seed coat epidermal cell differentiation is in progress. Steadystate mRNA levels are represented as relative expression compared to two reference genes, EF1aA4 and the seed reference gene (At4g12590). Error bars represent SE values from 3 biological replicates $(n=9)$. Statistical analyses were performed using a Wilcoxon test and comparing to the wild-type, ${ }^{*} p<0.05 \%,{ }^{* *} p<$ 0.01 . 

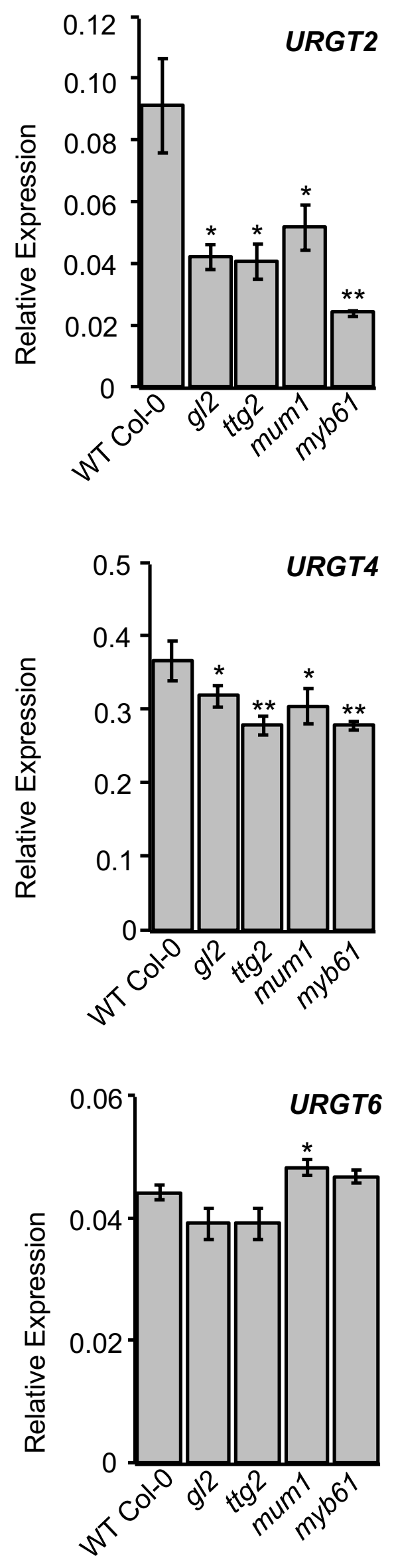

Figure 10: Modulation of URGT2, URGT4 and URGT6 expression by MUM1, MYB61, TTG1 and GL2 transcription regulators

Comparison of URGT2, URGT4 and URGT6 expression in developing seeds of the wild-type (WT Col-0) to g/2-6, ttg2-3, mum1/luh-3 and myb61 mutants reverse transcription quantitative PCR (RT-qPCR) analysis were used to determine the URGT2, URGT4 and URGT6 transcript levels in developing seeds at 8 days after pollination, which corresponds to the developmental stage when mucilage deposition is maximal in the wild-type. Steady state mRNA levels are represented as relative expression compared to two reference genes, EF1 $\alpha A 4$ and the seed reference gene (At4g12590). SE values from 3 biological replicates $(n=9)$. Statistical differences were analyzed using the Wilcoxon test and comparing to the wild-type, ${ }^{*} p<0.05 \%,{ }^{* *} p<0.01$. 


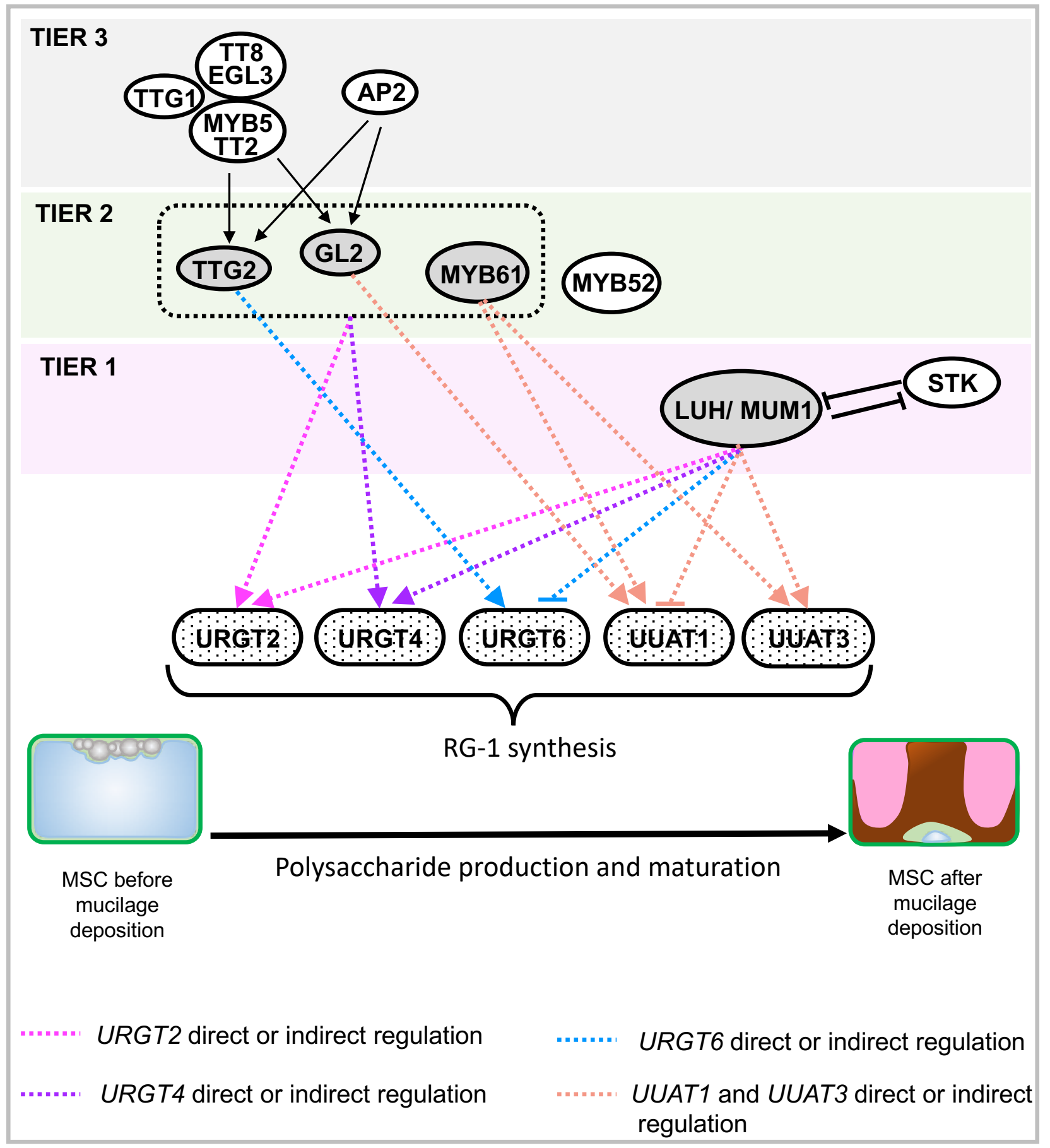

Figure 11: Hypothetical model of URGT2, URGT4, and URGT6 transcriptomic regulation by specific epidermal cell transcription factors

Expression levels observed in mutants indicate that TRANSPARENT TESTA GLABRA2 (TTG2), MYB61, GLABRA2 (GL2), and LEUNIG HOMOLOG/ MUCILAGE-MODIFIED1 (LUHI MUM1) transcriptional regulators directly or indirectly control the expression of URGT2, URGT4, URGT6, UUAT1 and UUAT3. URGT2, URGT4, and URGT6 transport UDP-Rha, one of the principal substrates of RG-I, and are independently required for the elongation of soluble mucilage RG-I polymers. UUAT1 and UUAT3 transport UDPUronic acid and undetermined UDP-Sugars that are also required for the synthesis of mucilage pectin (Saez-Aguayo et al., 2017; Parra-Rojas et al., 2019). URGT2, URGT6, URGT4, UUAT1, UUAT3, also act in adherent mucilage structuration. White proteins: Transcription factors described as acting on mucilage secretory cells (MSC) development. Doted proteins: UDP-Sugar Transporters regulated by Transcription Factors (gray proteins) evaluated in this study. Colored and dotted arrows indicate regulation demonstrated here. MSC, mucilage secretory cells. 


\section{Parsed Citations}

Alonso JM, Stepanova AN, Leisse TJ, Kim CJ, Chen H, Shinn P, Stevenson DK, Zmmerman J, Barajas P, Cheuk R, et al. 2003. Genomewide insertional mutagenesis of Arabidopsis thaliana. Science. 301, 653-657.

Google Scholar: Author Only Title Only Author and Title

Arsovski AA, Haughn GW, Western TL. 2010. Seed coat mucilage cells of Arabidopsis thaliana as a model for plant cell wall research. Plant Signaling \& Behaviour. 5, 796-801.

Google Scholar: Author Only Title Only Author and Title

Atmodjo MA, Hao Z, Mohnen D. 2013. Evolving Views of Pectin Biosynthesis. Annual Review of Plant Biology. 64, 747-779.

Google Scholar: Author Only Title Only Author and Title

Bassel GW, Fung P, Freeman Chow T-f, Foong JA, Provart NJ, Cutler SR. 2008. Elucidating the Germination Transcriptional Program Using Small Molecules. Plant Physiology. 147, 143-155.

Google Scholar: Author Only Title Only Author and Title

Blakeney AB, Harris PJ, Henry RJ, Stone BA 1983. A simple and rapid preparation of alditol acetates for monosaccharide analysis, Carbohydrate Researc. 113, 291-299.

Google Scholar: Author Only Title Only Author and Title

Brent LR, Malcolm AO, Debra M. 2001. Pectins: structure, biosynthesis, and oligogalacturonide-related signaling, Phytochemistry. 57, 929-967.

Google Scholar: Author Only Title Only Author and Title

Carpita, NC. and Gibeaut, DM. 1993. Structural models of primary cell walls in flowering plants: consistency of molecular structure with the physical properties of the walls during growth. The Plant Journal. 3, 1-30.

Google Scholar: Author Only Title Only Author and Title

Clough SJ and Bent AF. 1998. Floral dip: a simplified method for Agrobacterium mediated transformation of Arabidopsis thaliana. The Plant Journal.16: 735-743.

Google Scholar: Author Only Title Only Author and Title

Cosgrove DJ. 2016. Plant cell wall extensibility: connecting plant cell growth with cell wall structure, mechanics, and the action of wallmodifying enzymes, Journal of Experimental Botany. 67, 463-476.

Google Scholar: Author Only Title Only Author and Title

Dean GH, Zheng H, Tewari J, Huang J, Young DS, Hwang YT, Western TL, Carpita NC, McCann MC, Mansfield SD, Haughn GW. 2007. The Arabidopsis MUM2 gene encodes a $\beta$-galactosidase required for the production of seed coat mucilage with correct hydration properties. Plant Cell. 19, 4007-4021.

Google Scholar: Author Only Title Only Author and Title

Ebert B, Rautengarten C, Guo X, Xiong G, Stonebloom S, Smith-Moritz AM, Herter T, Chan LJ, Adams PD, Petzold CJ, et al. 2015. Identification and characterization of a Golgi-localized UDP-xylose transporter family from Arabidopsis. Plant Cell. 27, 1218-1227.

Google Scholar: Author Only Title Only Author and Title

Fabrissin I, Cueff G, Berger A, Granier F, Sallé C, Poulain D, Ralet M-C, North H.M et al. 2019. Natural variation reveals a key role for rhamnogalacturonan I in seed outer mucilage and underlying genes. Plant Physiol. pp.00763.2019. doi: 10.1104/pp.19.00763.

Google Scholar: Author Only Title Only Author and Title

Francoz E, Ranocha P, Burlat V, Dunand C. 2015. Arabidopsis seed mucilage secretory cells: regulation and dynamics. Trends Plant Science. 20, 515-524.

Google Scholar: Author Only Title Only Author and Title

Golz JF, Allen PJ, Li SF, Parish RW, Jayawardana NU, Bacic A et al. 2018. Layers of regulation - Insights into the role of transcription factors controlling mucilage production in the Arabidopsis seed coat. Plant Sci. 272, 179-192.

Google Scholar: Author Only Title Only Author and Title

Hong SM, Bahn SC, Lyu A, Jung HS, Ahn JH. 2010. Identification and testing of superior reference genes for a starting pool of transcript normalization in Arabidopsis. Plant Cell Physiology 51, 1694-1706.

Google Scholar: Author Only Title Only Author and Title

Huang J, DeBowles D, Esfandiari E, Dean G, Carpita NC, Haughn GW. 2011. The Arabidopsis transcription factor LUH/MUM1 is required for extrusion of seed coat mucilage. Plant Physiology. 156, 491-502.

Google Scholar: Author Only Title Only Author and Title

Hu R, Li J, Wang X, Zhao X, Yang X, Tang Q, He G, Zhou G, Kong Y. 2016. Xylan synthesized by Irregular Xylem 14 (IRX14) maintains the structure of seed coat mucilage in Arabidopsis. Journal of Experimental Botany. 67, 1243-1257.

Google Scholar: Author Only Title Only Author and Title

Ishida T, Hattori S, Sano R, Inoue K, Shirano Y, Hayashi H, Shibata D, Sato S, Kato T, Tabata S, et al. 2007. Arabidopsis TRANSPARENT TESTA GLABRA2 is directly regulated by R2R3 MYB transcription factors and is involved in regulation of GLABRA2 transcription in epidermal differentiation. Plant Cell. 19, 2531-2543. 
Google Scholar: Author Only Title Only Author and Title

Knappe S, Flügge Ul, Fischer K. 2003. Analysis of the plastidic phosphate translocator gene family in Arabidopsis and identification of new phosphate translocator-homologous transporters, classified by their putative substrate-binding site. Plant Physiology.131, 11781190.

Google Scholar: Author Only Title Only Author and Title

Kong Y, Zhou G, Abdeen AA, et al. 2013. GALACTURONOSYLT RANSFERASE-LIKE5 is involved in the production of Arabidopsis seed coat mucilage. Plant Physiology. 163: 1203-1217.

Google Scholar: Author Only Title Only Author and Title

Le BH, Cheng C, Bui AQ, Wagmaister JA, Henry KF, Pelletier J, Kwong L, Belmonte M, Kirkbride R, Horvath S, Drews GN, Fischer RL, Okamuro JK, Harada JJ, ... Goldberg RB. 2010. Global analysis of gene activity during Arabidopsis seed development and identification of seed-specific transcription factors. Proceedings of the National Academy of Sciences of the United States of America. 107(18), 806370.

\section{Google Scholar: Author Only Title Only Author and Title}

Levesque-Tremblay G, Pelloux J, Braybrook SA, Müller K. 2015. Tuning of pectin methylesterification: consequences for cell wall biomechanics and development. Planta. 242, 791-811.

Google Scholar: Author Only Title Only Author and Title

Macquet A, Ralet MC, Kronenberger J, Marion-Poll A, North HM. 2007a. In situ, chemical and macromolecular study of the composition of Arabidopsis thaliana seed coat mucilage. Plant Cell Physiology. 48: 984-999.

Google Scholar: Author Only Title Only Author and Title

Macquet A, Ralet MC, Loudet O, Kronenberger J, Mouille G, Marion-Poll, A, and North HM. 2007b. Anaturally occurring mutation in an Arabidopsis accession affects a beta-D-galactosidase that increases the hydrophilic potential of rhamnogalacturonan I in seed mucilage. Plant Cell. 19, 3990-4006.

Google Scholar: Author Only Title Only Author and Title

Mohnen D. 2008. Pectin structure and biosynthesis, Current Opinion in Plant Biology. 11, 266-277.

Google Scholar: Author Only Title Only Author and Title

Nakagawa T, Nakamura S, Tanaka K, Kawamukai M, Suzuki T, Nakamura K, Kimura T, Ishiguro S. 2008. Development of R4 Gateway Binary Vectors (R4pGWB) Enabling High-Throughput Promoter Swapping for Plant Research. Bioscience, Biotechnology, and Biochemistry. 72:2, 624-629.

Google Scholar: Author Only Title Only Author and Title

North HM, De Almeida A, Boutin J-P, Frey A, To A, Botran L, Sotta B, Marion-Poll A 2007. The Arabidopsis ABA deficient mutant aba4 demonstrates that the major route for stress-induced ABA accumulation is via neoxanthin isomers. Plant Journal 50, $810-824$.

Google Scholar: Author Only Title Only Author and Title

North HM, Berger A Saez-Aguayo S, Ralet M-C. 2014. Understanding polysaccharide production and properties using seed coat mutants: future perspectives for the exploitation of natural variants. Annals of Botany. 114: 1251-1263.

Google Scholar: Author Only Title Only Author and Title

Orellana A, Moraga C, Araya M, Moreno A 2016. Overview of nucleotide sugar transporter gene family functions across multiple species. Journal of Molecular Biology. 428, 3150-3165.

Google Scholar: Author Only Title Only Author and Title

Parra Rojas JP, Largo-Gosens A, Carrasco T, Celiz-Balboa J, Temple H, Sanhueza D, Reyes FC, Meneses C, Saez-Aguayo S, and Orellana A 2019. New steps in mucilage biosynthesis revealed by analysis of the transcriptome of UDP-rhamnose/UDP-galactose transporter 2 (URGT2) mutant. Journal of Experimental Botany. 70, 5071-5088.

Google Scholar: Author Only Title Only Author and Title

Ralet M-C, Tranquet O, Poulain D, Moïse A, Guillon F. 2010. Monoclonal antibodies to rhamnogalacturonan I backbone. Planta. 231, 1373-1383.

Google Scholar: Author Only Title Only Author and Title

Ralet M-C, Crépeau MJ, Vigouroux J, Tran J, Berger A, Sallé C, Granier F, Botran L, North HM. 2016. Xylans provide the structural driving force for mucilage adhesion to the Arabidopsis seed coat. Plant Physiology. 171, 165-178.

Google Scholar: Author Only Title Only Author and Title

Rasool B, McGowan J, Pastok D, Marcus SE, Morris J, Verrall SR, Hedley PE, Hancock RD, Foyer, CH. 2017. Redox control of aphid resistance through altered cell wall composition and nutritional quality. Plant Physiology.175, 259-271.

Google Scholar: Author Only Title Only Author and Title

Rautengarten C, Ebert B, Moreno I, Temple H, Herter T, Link B, Doñas-Cofré D, Moreno A, Saez-Aguayo S, Blanco F, et al. 2014. The Golgi localized bifunctional UDP- rhamnose/UDP-galactose transporter family of Arabidopsis. Proceedings of the National Academy of Sciences USA.111, 11563-11568.

Google Scholar: Author Only Title Only Author and Title

Reyes F, Orellana A 2008. Golgi transporters: opening the gate to cell wall polysaccharide biosynthesis. Current Opinion of Plant 
Biology. 11, 244-251.

Google Scholar: Author Only Title Only Author and Title

Saez-Aguayo S, Ralet M-C, Berger A, Botran L, Ropartz D, Marion-Poll A, North HM. 2013. PECTIN METHYLESTERASE INHIBITOR6 promotes Arabidopsis mucilage release by limiting methylesterification of homogalacturonan in seed coat epidermal cells. Plant Cell. 25, 308-323.

Google Scholar: Author Only Title Only Author and Title

Saez-Aguayo S, Rondeau-Mouro C, Macquet A, Kronholm I, Ralet MC, Berger A, Sallé C, Poulain D, Granier F, Botran L, Loudet O, de Meaux J, Marion-Poll A North HM. 2014. Local evolution of seed flotation in Arabidopsis. PLoS Genet. 2014;10(3):e1004221.

doi:10.1371/journal.pgen.1004221

Google Scholar: Author Only Title Only Author and Title

Saez-Aguayo S, Rautengarten C, Temple H, Sanhueza D, Ejsmentewicz T, Sandoval-Ibañez O, Doñas D, Parra-Rojas JP, Ebert B, Lehner A et al. 2017. UUAT1 is a Golgi-localized UDP-uronic acid transporter that modulates the polysaccharide composition of Arabidopsis seed mucilage. Plant Cell. 29. 129-143.

Google Scholar: Author Only Title Only Author and Title

Schindelin J, Ruede CT, Hiner MC, Eliceiri KW. 2015. The ImageJ ecosystem: An open platform for biomedical image analysis. Molecular reproduction and development. 82, 518-29.

Google Scholar: Author Only Title Only Author and Title

Shi D, Ren A, Tang X, Qi G, Xu Z, Chai G, Hu R, Zhou G, Kong Y. 2018. MYB52 negatively regulates pectin demethylesterification in seed coat mucilage. Plant Physiology. 176, 2737-2749.

Google Scholar: Author Only Title Only Author and Title

Sullivan, S., Ralet, M.-C., Berger, A, Diatloff, E., Bischoff, V., Gonneau, M., Marion-Poll, A, and North, H.M. 2011. CESA5 is required for the synthesis of cellulose with a role in structuring the adherent mucilage of Arabidopsis seeds. Plant Physiol. 156: $1725-1739$.

Google Scholar: Author Only Title Only Author and Title

Tan L, Eberhard S, Pattathil S, Warder C, Glushka J, Yuan C, Hao Z, Zhu X, Avci U, Miller J.S, Baldwin D, Pham C, Orlando R, Darvill A, Hahn M.G, Kieliszewski M.J , Mohnen D. 2013. An Arabidopsis Cell Wall Proteoglycan Consists of Pectin and Arabinoxylan Covalently Linked to an Arabinogalactan Protein. The Plant Cell Jan 2013, 25 (1) 270-287.

Google Scholar: Author Only Title Only Author and Title

Takenaka Y, Kato K, Ogawa-Ohnishi M, Tsuruhama K, Kajiura H, Yagyu K, Takeda A, Takeda Y, Kunueda T, Hara-Nishimura I, Kuroha T, Nishitani K, Matsubqyashi Y, Ishimizu T. 2018. Pectin RG-I rhamnosyltransferases represent a novel plant-specific glycosyltransferase family. Nat Plants. 4, 669-676.

Google Scholar: Author Only Title Only Author and Title

Takenaka Y, Watanabe Y, Schuetz M, Unda F, Hill JL, Phookaew P, Yoneda A, Mansfield SD, Samuels L, Ohtani M, Demura T. 2018. Patterned Deposition of Xylan and Lignin is Independent from that of the Secondary Wall Cellulose of Arabidopsis Xylem Vessels. The Plant Cell, 30, 2663-2676.

Google Scholar: Author Only Title Only Author and Title

Temple H, Saez-Aguayo S, Reyes FC, Orellana A 2016. The inside and outside: topological issues in plant cell wall biosynthesis and the roles of nucleotide sugar transporters. Glycobiology. 26, 913-925.

Google Scholar: Author Only Title Only Author and Title

Thibault, JE. 1979 .Automisation du dosage des subtances pectiques par la methode au meta-hidroxydiphenyl. Lebensm-Wiss.

Technol. 12, 247.

Verhertbruggen Y, Marcus SE, Chen J, Knox JP. 2013. Cell wall pectic arabinans influence the mechanical properties of Arabidopsis thaliana inflorescence stems and their response to mechanical stress. Plant Cell Physiology. 54, 1278-1288.

Google Scholar: Author Only Title Only Author and Title

Voiniciuc C, Yang B, Schmidt MH, Günl M, Usadel B. 2015a. Starting to gel: how Arabidopsis seed coat epidermal cells produce specialized secondary cell walls. Integrative Journal of Molecular Sciences. 16, 3452-3473.

Google Scholar: Author Only Title Only Author and Title

Voiniciuc C, Schmidt MH, Berger A, Yang B, Ebert B, Scheller HV, North HM, Usadel B, Günl M. 2015b. MUCILAGE-RELATED10 produces galactoglucomannan that maintains pectin and cellulose architecture in Arabidopsis seed mucilage. Plant Physiology. 169, 403-420.

Google Scholar: Author Only Title Only Author and Title

Voiniciuc C, Günl M, Schmidt MH, Usadel B. 2015c. Highly branched xylan made by IRREGULAR XYLEM14 and MUCILAGE-RELATED21 links mucilage to Arabidopsis seeds. Plant Physiology. 169, 2481-2495.

Google Scholar: Author Only Title Only Author and Title

Voiniciuc C, Engle KA, GünI M, Dieluweit S, Heinrich-Wilhelm MS,Yang JY, Moremen WK, Mohnen D, Usadel B. 2018. Identification of Key Enzymes for Pectin Synthesis in Seed Mucilage. Plant Physiology. 178, 1045-1064.

Google Scholar: Author Only Title Only Author and Title 
Walker M, Tehseen M, Doblin MS, Pettolino FA, Wilson SM, Bacic A, Golz JF. 2011. The transcriptional regulator LEUNIG_HOMOLOG regulates mucilage release from the Arabidopsis testa. Plant Physiology. 156: 46-60.

Google Scholar: Author Only Title Only Author and Title

Western TL, Skinner DJ, Haughn GW. 2000. Differentiation of mucilage secretory cells of the Arabidopsis seed coat. Plant Physiology. 122, 345-356.

Google Scholar: Author Only Title Only Author and Title

Western TL, Young DS, Dean GH, Tan WL, Samuels AL, Haughn GW. 2004. MUCILAGE-MODIFIED4 encodes a putative pectin biosynthetic enzyme developmentally regulated by APETALA2, TRANSPARENT TESTAGLABRA1, and GLABRA2 in the Arabidopsis seed coat. Plant Physiology 134, 296-306.

Google Scholar: Author Only Title Only Author and Title

Western TL. 2012. The sticky tale of seed coat mucilages: production, genetics, and role in seed germination and dispersal. Seed Science Research. 22, 1-25.

Google Scholar: Author Only Title Only Author and Title

Williams M, Cornuault V, Irani AH, Vaughan Symonds V, Malmstrom J, An Y, Sims IA, Carnachan SM, Sallé C, and North HM. 2020. Polysaccharide Structures in the Outer Mucilage of Arabidopsis Seeds Visualised by AFM. Biomacromolecules. DOI:

10.1021/acs.biomac.9b0175.

Google Scholar: Author Only Title Only Author and Title

Willats WG, McCarnety L, Mackie W, Knox JP. 2001. Pectin: cell biology and prospects for functional analysis. Plant Mol. Bio. 47, 9-27. Google Scholar: Author Only Title Only Author and Title

Winter D, Vinegar B, Nahal H, Ammar R, Wilson GV, Provart, NJ. 2007. An "Electronic Fluorescent Pictograph" browser for exploring and analyzing large-scale biological data sets. PloS one. 2(8), e718.

Google Scholar: Author Only Title Only Author and Title

Wolf S. 2017. Plant cell wall signalling and receptor-like kinases. Biochemical Journal. 474, 471-492.

Google Scholar: Author Only Title Only Author and Title

Young RE, McFarlane HE, Hahn MG, Western TL, Haughn GW, Samuels AL. 2008. Analysis of the Golgi apparatus in Arabidopsis seed coat cells during polarized secretion of pectin-rich mucilage. Plant Cell. 20, 1623-1638.

Google Scholar: Author Only Title Only Author and Title

Zhao X, Qiao L, Wu AM. 2017. Effective extraction of Arabidopsis adherent seed mucilage by ultrasonic treatment. Scientific Reports. 7, 40672.

Google Scholar: Author Only Title Only Author and Title 